\title{
Quantitative two-photon laser-induced fluorescence measurements of atomic hydrogen densities, temperatures, and velocities in an expanding thermal plasma
}

Citation for published version (APA):

Boogaarts, M. G. H., Mazouffre, S., Brinkman, G. J., Heijden, van der, H. W. P., Vankan, P. J. W., Mullen, van der, J. J. A. M., Schram, D. C., \& Döbele, H. F. (2002). Quantitative two-photon laser-induced fluorescence measurements of atomic hydrogen densities, temperatures, and velocities in an expanding thermal plasma. Review of Scientific Instruments, 73(1), 73-86. https://doi.org/10.1063/1.1425777

DOI:

10.1063/1.1425777

Document status and date:

Published: 01/01/2002

Document Version:

Publisher's PDF, also known as Version of Record (includes final page, issue and volume numbers)

Please check the document version of this publication:

- A submitted manuscript is the version of the article upon submission and before peer-review. There can be important differences between the submitted version and the official published version of record. People interested in the research are advised to contact the author for the final version of the publication, or visit the $\mathrm{DOI}$ to the publisher's website.

- The final author version and the galley proof are versions of the publication after peer review.

- The final published version features the final layout of the paper including the volume, issue and page numbers.

Link to publication

\footnotetext{
General rights

- You may freely distribute the URL identifying the publication in the public portal. follow below link for the End User Agreement:

www.tue.nl/taverne

Take down policy

If you believe that this document breaches copyright please contact us at:

openaccess@tue.nl

providing details and we will investigate your claim.
}

Copyright and moral rights for the publications made accessible in the public portal are retained by the authors and/or other copyright owners and it is a condition of accessing publications that users recognise and abide by the legal requirements associated with these rights.

- Users may download and print one copy of any publication from the public portal for the purpose of private study or research.

- You may not further distribute the material or use it for any profit-making activity or commercial gain

If the publication is distributed under the terms of Article $25 \mathrm{fa}$ of the Dutch Copyright Act, indicated by the "Taverne" license above, please 


\title{
Quantitative two-photon laser-induced fluorescence measurements of atomic hydrogen densities, temperatures, and velocities in an expanding thermal plasma
}

\author{
M. G. H. Boogaarts, S. Mazouffre, G. J. Brinkman, H. W. P. van der Heijden, P. Vankan, ${ }^{\text {a) }}$ \\ J. A. M. van der Mullen, and D. C. Schram \\ Department of Applied Physics, Eindhoven University of Technology, P.O. Box 513, 5600 MB Eindhoven, \\ The Netherlands \\ H. F. Döbele \\ Institut für Laser- und Plasmaphysik, Universität GH Essen, 45117 Essen, Germany
}

(Received 9 August 2001; accepted for publication 15 October 2001)

\begin{abstract}
We report on quantitative, spatially resolved density, temperature, and velocity measurements on ground-state atomic hydrogen in an expanding thermal $\mathrm{Ar}-\mathrm{H}$ plasma using two-photon excitation laser-induced fluorescence (LIF). The method's diagnostic value for application in this plasma is assessed by identifying and evaluating the possibly disturbing factors on the interpretation of the LIF signal in terms of density, temperature, and velocity. In order to obtain quantitative density numbers, the LIF setup is calibrated for $\mathrm{H}$ measurements using two different methods. A commonly applied calibration method, in which the LIF signal from a, by titration, known amount of $\mathrm{H}$ generated by a flow-tube reactor is used as a reference, is compared to a rather new calibration method, in which the $\mathrm{H}$ density in the plasma jet is derived from a measurement of the two-photon LIF signal generated from krypton at a well-known pressure, using a known $\mathrm{Kr}$ to $\mathrm{H}$ detection sensitivity ratio. The two methods yield nearly the same result, which validates the new $\mathrm{H}$ density calibration. Gauging the new "rare gas method" by the "flow-tube reactor method," we find a krypton to hydrogen two-photon excitation cross section ratio $\sigma_{\mathrm{Kr}}^{(2)} / \sigma_{\mathrm{H}}^{(2)}$ of 0.56 , close to the reported value of 0.62 . Since the $\mathrm{H}$ density calibration via two-photon LIF of krypton is experimentally far more easy than the one using a flow-tube reactor, it is foreseen that the "rare gas method" will become the method of choice in two-photon LIF experiments. The current two-photon LIF detection limit for $\mathrm{H}$ in the $\mathrm{Ar}-\mathrm{H}$ plasma jet is $10^{15} \mathrm{~m}^{-3}$. The accuracy of the density measurements depends on the accuracy of the calibration, which is currently limited to $33 \%$. The reproducibility depends on the signal-to-noise $(\mathrm{S} / \mathrm{N})$ ratio in the LIF measurements and is orders of magnitude better. The accuracy in the temperature determination also depends on the S/N ratio of the LIF signal and on the ratio between the Doppler-width of the transition and the linewidth of the excitation laser. Due to the small $\mathrm{H}$ mass, the current linewidth of the UV laser radiation is never the accuracy limiting factor in the $\mathrm{H}$ temperature determination, even not at room temperature. Quantitative velocity numbers are obtained by measuring the Doppler shift in the $\mathrm{H}$ two-photon excitation spectrum. Both the radial and axial velocity components are obtained by applying a perpendicular and an antiparallel excitation configuration, respectively. The required laser frequency calibration is accomplished by simultaneously recording the $\mathrm{I}_{2}$ absorption spectrum with the fundamental frequency component of the laser system. This method, which is well-established in spectroscopic applications, enables us to achieve a relative accuracy in the transition frequency measurement below $10^{-6}$, corresponding to an accuracy in the velocity of approximately $200 \mathrm{~m} / \mathrm{s}$. This accuracy is nearly laser linewidth limited. (C) 2002 American Institute of Physics. [DOI: 10.1063/1.1425777]
\end{abstract}

\section{INTRODUCTION}

When a thermal plasma created by a cascaded arc expands through a nozzle into a low pressure region, a versatile high-quality particle beam is obtained that has been demonstrated to have many interesting features. ${ }^{1,2}$ Such an expanding thermal plasma (ETP) has important applications in plasma processing, as, for instance, in plasma enhanced chemical vapor deposition, surface passivation, and etching.

${ }^{a)}$ Corresponding author; electronic mail: p.j.w.vankan@tue.nl
Particularly the application in thin layer deposition of hydrogenated amorphous silicon $(a-\mathrm{Si}: \mathrm{H})$ and carbon $(a-\mathrm{C}: \mathrm{H})$, where deposition of good quality material has been demonstrated at rates up to $10 \mathrm{~nm} / \mathrm{s},{ }^{2,3}$ is very efficient. In many of these plasma processing applications, atomic hydrogen plays a key role as a chemical agent, and it is, therefore, often an important constituent of the plasma beams that are generated for those applications.

True control and optimization of the applications of an expanding plasma beam demands a thorough knowledge and understanding of the gas-phase plasma composition and ki- 
netics as well as of the processes taking place at the interface between plasma and condensed material. The study of the ETP features, and of low-temperature plasmas in general, requires a technique that is nonintrusive, in situ, and speciesselective. In addition, the technique should give quantitative results, and when it is applied for the detection of reactive (and thus short-lived) species, it should have a sensitivity that is sufficiently high to detect the consequently low density of such species. Optical/spectroscopic techniques generally are nonintrusive, in situ (and remote), and speciesselective, and the laser-induced fluorescence (LIF) technique adds an extreme sensitivity and an excellent spatial and temporal resolution to that. Numerous publications show the high diagnostic value of LIF as a probe for small molecules, molecular radicals, and atoms in their ground or excited states, in which easy concepts are used to interpret the LIF signal in terms of density, temperature, and velocity of the probed species. Consequently, the technique has found widespread application in many research areas including lowtemperature plasma research. ${ }^{4}$

When applying LIF (and any other active optical/ spectroscopic technique) for the detection of ground-state atoms, one has to deal with the large energy spacing between the ground state and any excited electronic state in these atoms. In the case of atomic hydrogen, the energy difference between the $n=1$ ground state and the $n=2$ state is $10.2 \mathrm{eV}$, corresponding to a $121.6 \mathrm{~nm}$ Lyman- $\alpha$ photon, which is well in the vacuum ultraviolet (VUV) wavelength range. The use of a single-photon excitation LIF scheme for ground-state atoms has some serious drawbacks. First of all one needs experimentally demanding techniques for the generation of the necessary VUV photons. Moreover, many of the investigated media will be optically thick for these VUV photons due to a large single-photon absorption cross section and/or a relatively high ground-state atom density. These problems can be avoided by using a two-photon excitation scheme.

Such a two-photon excitation scheme was first demonstrated for atomic hydrogen and deuterium by Bokor et al. in $1981 .{ }^{5}$ In a short period of time two-photon excitation laserinduced fluorescence detection of ground-state atoms was also reported for nitrogen ${ }^{6}$ oxygen, ${ }^{6-8}$ chlorine, ${ }^{9}$ sulfur, ${ }^{10}$ carbon, ${ }^{11}$ and fluorine. ${ }^{12,13}$ After this pioneering work in which the measurement principle for these species was demonstrated, the two-photon LIF technique has been successfully applied to study a variety of reactive environments. In the field of low-temperature plasma research, where the technique is also referred to as "two-photon absorption laser induced fluorescence (TALIF)," there has been a considerable effort on atomic hydrogen detection, with important contributions from the group of Miller ${ }^{14-18}$ and many others. ${ }^{19-23}$

In these studies, the H LIF signal from the plasma is used to determine the hydrogen concentration, assuming that the LIF signal intensity is proportional to the ground-state density. The valuable density information gained in these experiments, however, is usually only qualitative in nature, due to one of the few disadvantages of the LIF technique. Unless an analytical expression for the relation between the LIF signal intensity and the density of the probed species can be derived that can be evaluated from first principles, quan- titative density measurements require a calibration of the LIF setup. In the case of stable neutral atoms or molecules a calibration is easily accomplished with, e.g., a gas cell containing a well-known pressure of the probed species. In the case of short-lived species like radicals or ions, calibration is less trivial.

The urgent need of quantitative data in this and other research fields has led to the proposal and/or demonstration of a few calibration methods for (two-photon) LIF measurements of ground-state atom densities. These methods can be distinguished by their different approach. The most common approach is to use a known amount of the probed species from an independent source as a reference that is subsequently measured in the same measurement setup. In this approach often a "flow-tube reactor" is used as a source of ground-state atomic radicals, in which the concentration of these radicals is determined by a chemical titration. This method is based on well-established flow reactor and titration techniques in chemistry ${ }^{24}$ and has been applied for the calibration of LIF density measurements of several atomic radicals in plasmas, including atomic hydrogen ${ }^{17,25}$ and atomic oxygen. ${ }^{26}$

Another approach is to also use an independent quantitative measurement technique to determine the density of the probed species. An example of such an approach is to compare the LIF measurements on a line through the investigated medium with the line-of-sight integrated absorption by the medium. Since this approach requires the availability of two independent accurate measurement techniques, it is less commonly reported. ${ }^{20}$

A third and rather new approach is to generate a LIF signal from a known amount of a different, stable species by an excitation scheme that is very close to the one applied for the atomic radical. The atomic radical density in the plasma can then be determined from the ratio of the LIF signals when the relative detection sensitivity for the two species is known. This approach avoids the cumbersome generation of a known amount of atomic radicals from an independent source. The method has recently been demonstrated for the calibration of ground-state $\mathrm{H}$ densities ${ }^{27}$ as well as of ground-state $\mathrm{N}$ densities ${ }^{27,28}$ using a two-photon transition in krypton. The method has also been applied to calibrate the fluorescence yield of oxygen atoms via a two-photon transition in xenon. ${ }^{29}$ This method is quite elegant and experimentally easy compared to others, but it relies on critical excitation cross section data not yet confirmed by other experiments.

The LIF technique also enables the determination of the temperature of the probed species, which is an important parameter in plasma research since it characterizes the plasma and it determines the plasma kinetics. In the case of molecules, the internal (rotational and/or vibrational) temperature can be derived from the relative distribution over internal quantum states. In the case of atoms, this concept can only be used to determine an "electronic temperature," which often has a poor correlation with the kinetic temperature. The kinetic temperature can instead be derived from the velocity spread of the atoms, which is reflected in the Doppler width of the laser-induced transition. Of course this 
simple measurement concept only applies when the linewidth of the exciting radiation is of the same order or smaller than the Doppler width. For light atoms in reactive environments (like $\mathrm{H}$ in plasmas) this is often the case, thus enabling a reasonably accurate determination of the kinetic temperature of these atoms. ${ }^{16}$

Besides determining the velocity spread, and from that the temperature, of the particles from the Doppler width, the Doppler shift of the center frequency of the laser-induced transition can be used to determine the average velocity of these particles. The Doppler shift can be determined either in an absolute way, i.e., on an absolute frequency scale, in which case the laser frequency needs to be calibrated, or in a relative way by comparing the signal of the moving particles to that of a nonmoving reference. In the field of lowtemperature plasma research, the velocity and the corresponding kinetic energy of plasma particles is an important parameter, for instance, to determine particle and/or energy fluxes in plasma processing. Nevertheless, only a few studies exist in which the LIF technique is applied to measure velocities of particles in plasmas, ${ }^{30,31}$ and to the best of our knowledge our group was the first to report velocity measurements of ground-state atoms using the two-photon LIF technique. ${ }^{32}$

In this study, two-photon excitation laser-induced fluorescence is applied to perform quantitative and spatially resolved measurements of the density, temperature, and velocity of ground-state hydrogen atoms in an expanding thermal $\mathrm{Ar}-\mathrm{H}$ plasma created by a cascaded arc. The excitation and detection scheme for ground-state $\mathrm{H}$ applied in this work is the scheme that was originally demonstrated by Bokor et al. in $1981 .^{5}$ In this scheme $\mathrm{H}$ is excited with two $205 \mathrm{~nm}$ photons from the $n=1$ ground state to the $n=3$ state. The resulting nonresonant laser-induced fluorescence on the Balmer- $\alpha$ transition between the $n=3$ and $n=2$ state is detected at $656 \mathrm{~nm}$. After a description of the experimental setup in Sec. II, this scheme and its suitability for application in the ETP is discussed in detail in Sec. III. Next, the applied LIF measurement concept is described, and the determination of the local density, temperature, and velocity of $\mathrm{H}$ from the acquired LIF signal is discussed separately in the subsequent sections IV, V, and VI, respectively. Since we aim at accurate quantitative measurements, these sections give a careful analysis of the phenomena that (could) affect the interpretation of the LIF signal in terms of these quantities.

Quantitative velocity measurements are obtained by calibrating the laser frequency. This is accomplished by using a part of the output of the laser system at the fundamental frequency around $615 \mathrm{~nm}$ for the simultaneous recording of the molecular iodine absorption spectrum. In this region, the $\mathrm{I}_{2}$ spectrum shows many absorption lines that are tabulated in frequency to a high accuracy. ${ }^{33}$ This frequency calibration method is widely used in spectroscopic applications and it allows us to reach an accuracy in the velocity measurements that is sufficient to gain valuable information about the flow pattern in the ETP.

For the calibration of the LIF setup for the $\mathrm{H}$ density measurements, two different approaches are used. Section VII A describes the density calibration via the "classical" approach of a measurement of the H LIF signal from a flowtube reactor. This method is experimentally quite elaborate and needs careful execution. Therefore we also performed a calibration via the above mentioned "new" approach of generating a two-photon LIF signal from krypton at a fixed pressure, which is the subject of Sec. VIIB. The subsequent comparison between the two calibration methods enables a first validation of the previously reported excitation cross section data. ${ }^{27}$

The two-photon LIF technique has been used to completely map the $\mathrm{H}$ density, temperature, and velocity (along two independent axes) in the ETP. A lot of effort has been spent to quantify the data and to assess its reliability. Among others a time-resolved study has been performed, both experimentally and computationally, to investigate the occurrence and the effect of collisional quenching of the twophoton LIF signal in our experiments. All this resulted in a large data set from which a lot of physical information can be extracted. It enables, for instance, a detailed study of the expansion properties of the plasma jet. Here we focus on the basic measurement method, its applicability in the type of plasma we are studying, and on the techniques to quantify the data. The time-resolved study on collisional quenching of the two-photon LIF signal from low-temperature plasmas is reported in a separate paper, ${ }^{34}$ as is the in-depth physical discussion of the expansion properties of the plasma jet. ${ }^{32,35}$

\section{EXPERIMENTAL SETUP}

A scheme of the experimental setup is depicted in Fig. 1. The cascaded arc plasma source has already been described in detail elsewhere. ${ }^{36}$ In the experiments described in this article it is operated on a $40 \mathrm{~A} \mathrm{dc}$ current with a typical corresponding voltage of $100 \mathrm{~V}$ between the three cathodes and the anode plate. The plasma expands from the $3 \mathrm{~mm}$ diameter arc channel, where the pressure is typically 0.6 bar, into a roots-blower pumped vacuum vessel with a background pressure of $14 \mathrm{~Pa}$. For the atomic hydrogen measurements a 6 to $1 \mathrm{Ar}-\mathrm{H}_{2}$ gas mixture with a $50 \mathrm{sccs}$ Ar flow and a $8.3 \mathrm{sccs} \mathrm{H}_{2}$ flow is fed into the cascaded arc. These experimental conditions are close to those used for the fast deposition of $a$-Si:H. ${ }^{2}$ The cascaded arc plasma source is mounted on a translation stage. Spatial scans through the expanding plasma are performed by moving the cascaded arc relative to the intersection of the laser beam and the detection volume.

The laser system that is used to produce the tunable UV radiation is based on a pulsed Nd:YAG/dye-laser combination. The frequency-doubled output $(300 \mathrm{~mJ}$ pulse energy at $532 \mathrm{~nm}$ ) of a $50 \mathrm{~Hz}$ injection-seeded Nd:YAG laser (SpectraPhysics/Quanta-Ray, GCR-230) is used to pump a tunable dye laser (Spectra-Physics/Quanta-Ray, PDL-3). The dye laser has a bandwidth of $0.07 \mathrm{~cm}^{-1}$ and is used around 615 $\mathrm{nm}$. The output of the dye laser (typically $60 \mathrm{~mJ}$ ) is frequency-tripled in two stages. First, the fundamental dyelaser frequency is doubled in a KD*P crystal and then this frequency-doubled UV light is mixed with the residual red dye-laser output in a BBO crystal for sum-frequency generation. In between the $\mathrm{KD}^{*} \mathrm{P}$ and the $\mathrm{BBO}$ crystal a wave plate 


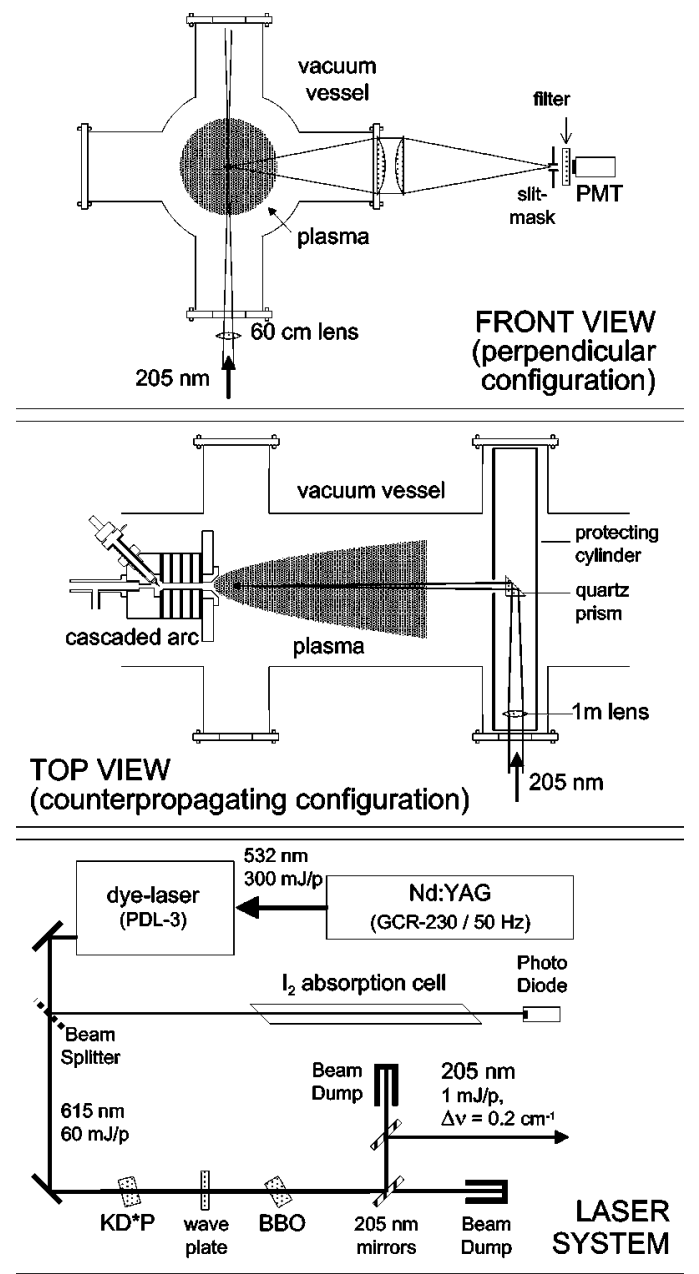

FIG. 1. Scheme of the experimental setup. The cascaded arc plasma source is mounted on a translation stage that enables spatially resolved measurements. The laser beam is directed either perpendicular to the expansion axis (as in the front view) or counterpropagating with respect to the expansion (as in the top view), depending on the required velocity measurement. The fluorescence is detected under right angles. Part of the dye-laser output is used for the simultaneous recording of the $\mathrm{I}_{2}$ absorption spectrum for wavelength calibration.

( $\lambda / 4$ for the blue beam) is used to match the polarization angles of the fundamental and the frequency-doubled light to each other again. The tripled frequency component is separated from the others and directed towards the vacuum vessel using high-power, high-reflective dielectric mirrors for 205 $\mathrm{nm}\left(45^{\circ}\right)$ that are transparent for the other wavelengths (LaserOptik $\mathrm{GmbH}$ ). Wavelength separation is in this way less perfect compared to, for instance, using Pellin-Broca prisms, but due to absorption losses with the latter method, the use of the mirrors is found to easily enhance the $205 \mathrm{~nm}$ output energy by a factor of 2 . This procedure results in typically 1 $\mathrm{mJ}$ of tunable UV light around $205 \mathrm{~nm}$, with an estimated bandwidth of $0.2 \mathrm{~cm}^{-1}$. Both crystals are automatically tracked while tuning the wavelength of the dye laser.

The frequency-tripled laser light is directed into the vessel either perpendicular to the plasma expansion axis or counterpropagating with respect to the expansion, depending on the required velocity measurement. In the default perpendicular configuration, where radial velocities can be measured, the UV laser light is focused into the plasma with a 60 $\mathrm{cm}$ lens. When axial velocities are to be measured, a $1 \mathrm{~m}$ focusing lens is used in combination with a quartz rightangle prism which is at a distance of at least $65 \mathrm{~cm}$ from the cascaded arc nozzle. The prism directs the light antiparallel to the plasma jet. In this way only the prism is exposed to the plasma, and the combination of its relatively large distance from the plasma source with its mounting in a water-cooled protection cylinder ensures a few hours of measurement time before cleaning of the prism is necessary.

The laser-induced fluorescence originating from the focus is imaged onto a slit mask, in a direction perpendicular to the plane formed by the laser beam and the symmetry axis of the expansion. This is done by two $10 \mathrm{~cm}$ diameter planoconvex lenses with focal lengths of $40 \mathrm{~cm}$. The $40 \mathrm{~cm}$ distance of the first lens from the laser focus is imposed by the geometry of the vessel. As a consequence, the solid angle of the detection is limited to $4.9 \times 10^{-2} \mathrm{sr}$. The slit mask, which is $0.35 \mathrm{~mm}$ wide and has a variable length up to $10 \mathrm{~mm}$, is placed parallel to the laser beam and defines the spatial resolution. The width of the slit mask ensures that all $\mathrm{H}$ fluorescence will pass the mask up to the highest observed $\mathrm{H}$ velocity. For a velocity of $4500 \mathrm{~m} / \mathrm{s}$ (see Sec. VI), the transit time through the detection volume is $\approx 80 \mathrm{~ns}$, which is nearly one order of magnitude longer than the $\mathrm{H}(n=3)$ fluorescence lifetime (10 ns, see Sec. IV). The laser-induced fluorescence that passes the slit mask is detected by a photomultiplier tube (PMT). For almost all LIF measurements a highly sensitive, gated PMT is used (Hamamatsu, R928). The continuous background light emitted by the plasma is strongly reduced by a Balmer- $\alpha\left(\mathrm{H}_{\alpha}\right)$ bandpass filter with a $10 \mathrm{~nm}$ bandwidth [full width at half maximum (FWHM)] centered around $656 \mathrm{~nm}$ in front of the PMT. However, the suppression of the plasma emission by the optical filter is not always sufficient to allow the PMT to be used with maximum gain. This is, for instance, the case in the $\mathrm{Ar}-\mathrm{H}$ plasma close to the nozzle orifice where the plasma emits much Balmer- $\alpha$ radiation. This is a well-known problem in the application of the LIF technique in light emitting media like those encountered in plasma and combustion research, which is usually solved by gating the detection. The Hamamatsu PMT R928 is equipped with a gate unit that enables triggered operation of the PMT during a short time interval with a preset duration between 0.8 and $10 \mu \mathrm{s}$. This further reduces the continuous background by a factor of $2 \times 10^{4}$, in principle enabling operation of the PMT with maximum gain in all circumstances. In the case of the Kr LIF measurements (see Sec. VII B) a long-pass filter that cuts off below $700 \mathrm{~nm}$ is used to reduce the laser stray light impinging onto the PMT.

For time-resolved fluorescence measurements a very fast "metal package" photomultiplier tube (Hamamatsu R5600P01) is used. ${ }^{34}$ This rather new type of PMT combines a $1.5 \mathrm{~ns}$ FWHM single photon response time with a gain of up to $10^{6}$. The PMT signal is recorded by a $500 \mathrm{MHz} 1 \mathrm{Gs} / \mathrm{s}$ 8-bit digital sampling oscilloscope. The signal trace is transferred to a PC where it can be analyzed. This enables both timeintegrated and time-resolved measurements. In the case of time-integrated measurements, it allows for baseline corrections and a precise signal integration over time, yielding a 


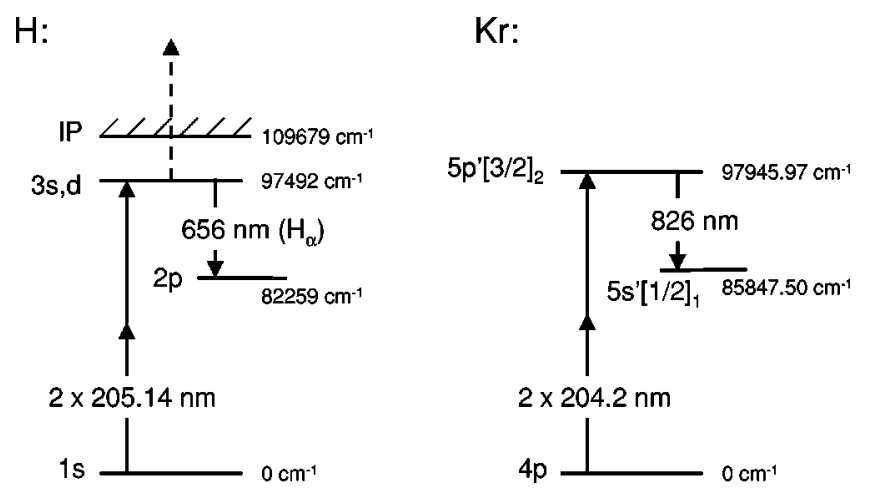

FIG. 2. Energy level schemes for the two-photon excitation laser-induced fluorescence measurements of ground-state hydrogen atoms (left) and ground-state krypton atoms (right).

signal-to-noise ratio better than those of analog integration devices, especially in the case of a high background signal. The PC also controls the wavelength scanning of the dye laser.

The laser wavelength is calibrated by recording simultaneously the $\mathrm{I}_{2}$ absorption spectrum, of which the line positions are tabulated to a high accuracy ${ }^{33}$ For this, part of the red dye-laser output is directed through a $\mathrm{I}_{2}$ absorption cell. This glass cell has a $2.5 \mathrm{~cm}$ diameter and a $60 \mathrm{~cm}$ length and contains only iodine (solid and vapor). The transmitted light intensity is measured behind the cell with a photodiode. The length of the cell is chosen to ensure easily measurable absorptions at room temperature. The absorption can be optimized by adjusting the $I_{2}$ vapor pressure with a heating wire around the cell.

\section{EXCITATION AND DETECTION OF GROUND-STATE ATOMIC HYDROGEN}

The excitation and detection scheme for ground-state atomic hydrogen applied in this work is the one that was first demonstrated by Bokor et al. in 1981. ${ }^{5}$ In this scheme (depicted on the left in Fig. 2) $\mathrm{H}$ is excited with two $205.14 \mathrm{~nm}$ photons from the $1 s^{2} S_{1 / 2}$ ground state to the $n=3$ level. The $n=3$ fine-structure sublevels cannot be resolved within the bandwidth of the $205 \mathrm{~nm}$ laser radiation $\left(\approx 0.2 \mathrm{~cm}^{-1}\right)$. As a result, in a spectral scan over the two-photon transition both the $3 s^{2} S_{1 / 2}$ and the $3 d^{2} D_{j}$ states will be excited, according to the two-photon selection rules $\Delta l=0, \pm 2$. The excitation is monitored by detection of the nonresonant fluorescence on the Balmer- $\alpha$ transition at $656.3 \mathrm{~nm}$ from the $3 d$ and $3 s$ states to the $2 p^{2} P_{j}$ states. Usually it is mentioned that the $3 d \leftarrow 1 s$ transition dominates over the $3 s \leftarrow 1 s$ transition due to a 7.56 times higher two-photon absorption cross section, ${ }^{5,19,20}$ but actually it depends on the degree of saturation of the transition whether the relative population of $3 d$ and $3 s$ is determined by the relative transition strength or rather by the ratio of the statistical weights of the respective quantum states. Moreover, we found evidence for a pure statistical distribution of the population over all the $n=3$ sublevels from an accurate measurement of the lifetime of the excited state,$^{34}$ even though the applied laser intensity assures that the transition is far from saturation (see Fig. 3). This can be explained by a complete $l$ mixing of the quantum

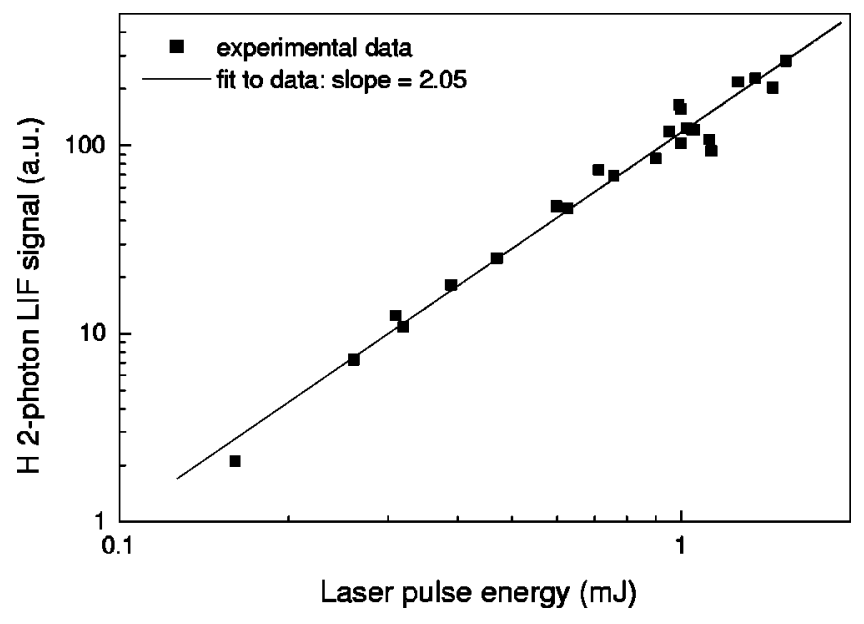

FIG. 3. Two-photon excitation laser-induced fluorescence signal of atomic hydrogen as a function of the $205 \mathrm{~nm}$ laser pulse energy. The solid line is a fit to the experimental data. It clearly shows that in our experimental setup the H LIF signal depends quadratically on the laser intensity over the entire range of used laser pulse energies.

states in an electric field. Thus far we have not been able to unambiguously establish the origin of this electric field, but since the $n=3$ lifetime and thus the degree of $l$ mixing does not vary within the plasma jet, it is likely to be the electric field associated with the propagating laser beam.

The scheme described here has some distinct advantages over various other schemes that have been proposed and/or demonstrated. First of all, two-photon excitation avoids some of the problems connected with single-photon excitation, as was mentioned in the Introduction. In addition, when exciting to $n=3$ or higher, fluorescence detection can be performed on a transition back to a state other than the ground state, which will significantly relax the need for suppression of stray light from the laser beam. Several schemes for twophoton excitation of $\mathrm{H}$ to $n=3$ and $n=4$ with detection of nonresonant fluorescence have been directly compared to each other. ${ }^{19}$ These schemes differ in the number of laser beams and the wavelength combinations used for excitation, the intensity, polarization, and bandwidth of the laser beams, and the wavelength of the fluorescence light. This detailed comparison shows that the above described scheme for twophoton excitation of $\mathrm{H}$ with a pulsed, narrow-band $205 \mathrm{~nm}$ laser beam gives the largest average number of fluorescence photons per incident laser photon.

Another characteristic of the applied $\mathrm{H}$ excitation scheme is that only one additional laser photon is needed for ionization of the excited atom, as is indicated in Fig. 2. When the laser intensity is increased, this ionization pathway will eventually limit the fluorescence yield, as the rate of $n=3$ depopulation via this process starts to compete with the radiative depopulation rate. This picture can be quantified by applying a rate-equation model for the laser-excited state population, as is, for instance, done in Ref. 19. This shows that for the $\mathrm{H}$ excitation scheme described here "the average number of fluorescence photons emitted per ground-state atom" saturates at $\approx 0.1$ for a laser intensity around $10^{9} \mathrm{~W} / \mathrm{cm}^{2}$. Usually, as is the case in the experiments described in this article, the laser intensity is kept at least one 
order of magnitude lower, thereby limiting the total number of particles that emit fluorescence photons to a few percent. Figure 3 shows the two-photon LIF signal of atomic hydrogen from the expanding $\mathrm{Ar}-\mathrm{H}$ plasma as a function of the laser intensity. For low laser intensities a quadratic dependence of this signal on the laser intensity should be observed, saturating to a lower order dependence for higher intensities. The slope of the data points in the log-log plot indeed equals 2 within the experimental accuracy, from which it can be concluded that the $\mathrm{H}$ two-photon excitation does not reach saturation in our experiment and that the LIF signal remains in the quadratic regime over the entire range of laser pulse energies used in our measurements.

Although keeping the laser intensity low, and thereby keeping the LIF signal in the quadratic regime, seems to be a safe situation at first sight, it needs a closer look. Average fluorescence photon numbers per ground-state atom of a few percent indicate relative excited state populations of the same order or higher, and a significant part of this population will end up as an ion-electron pair. In fact, one additional laser photon is sufficient to ionize not only the laser-excited atoms, but all atoms in excited states. Depending on the type of plasma and the ionization degree, these laser-induced excited state, ion, and electron densities can be several orders of magnitude higher than the respective stationary densities. In such a case, the applied laser diagnostic method can hardly be qualified as "nonintrusive." A more complete model is then needed to quantitatively assess the effect of the pulsed laser excitation on the local plasma parameters. This has been investigated in another study concurrently performed in our group. ${ }^{34}$ In this study, a time-resolved "collisional radiative model (CRM)" for $\mathrm{H}$ has been developed that models the response of a system of levels to a temporary disturbance of the level population distribution by, for instance, pulsed laser excitation of one of the levels. The study also deals with the implications for the observed signals and their interpretation. One of the conclusions is that, for conditions typically encountered in the ETP, the ion and electron densities can indeed temporarily increase easily by a factor of 5. Consequently, depending on the exact local plasma conditions, the two-photon LIF signal might suffer from "selfquenching," i.e., quenching of the laser-excited states by electrons that were created through laser-induced ionization. The issue of fluorescence quenching in the ETP is addressed in Sec. IV.

The above described $\mathrm{H}$ excitation and detection scheme is used to map the ground-state $\mathrm{H}$ distribution in the expanding plasma jet. To achieve this, two-photon LIF measurements are performed at various axial and radial positions. All LIF measurements are acquired by tuning the excitation laser and recording the resulting spectral profile of the two-photon absorption. In the case of velocity measurements, the $\mathrm{I}_{2}$ absorption spectrum is recorded simultaneously. This will be described in more detail in Sec. VI. As an example, Fig. 4 shows a spectral scan that is recorded in the expanding $\mathrm{Ar}-\mathrm{H}$ plasma at an axial position $z=50 \mathrm{~mm}$ on the centerline of the expansion (radial position $r=0 \mathrm{~mm}$ ). The $\mathrm{H}$ fluorescence signal is for all spectral scans fitted to a Gaussian profile. No non-Gaussian profiles have been observed. From these fits

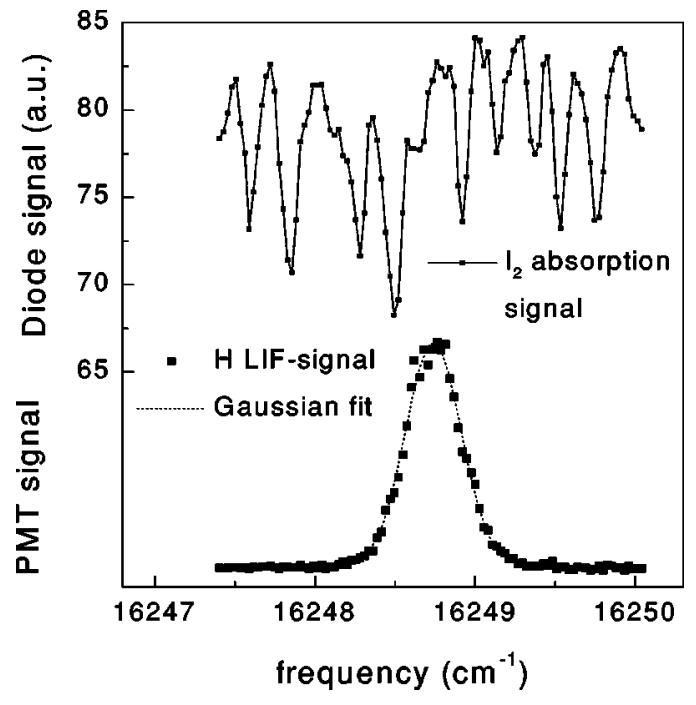

FIG. 4. Example of a spectral scan with simultaneous recording of the $\mathrm{H}$ two-photon LIF signal (PMT signal, lower trace) and the $\mathrm{I}_{2}$ absorption signal (diode signal, upper trace). The scan is recorded in the $\mathrm{Ar}-\mathrm{H}$ plasma jet at an axial position $z=50 \mathrm{~mm}$ on the centerline of the expansion (radial position $r=0 \mathrm{~mm}$ ). The horizontal axis is in units of the fundamental dyelaser frequency. This axis is calibrated by the position of the $\mathrm{I}_{2}$ absorption lines. To obtain the $\mathrm{H}$ transition frequency this scale has to be multiplied by a factor of 6 . The H LIF signal is fitted to a Gaussian profile and from this fit the local $\mathrm{H}$ density, temperature, and velocity are determined.

three local quantities are obtained. The local (relative) density is obtained from the integrated intensity (i.e., the area under the curve), while the local temperature is derived from the Doppler width of the spectral profile. In addition, the component of the velocity in the direction of the laser is determined from the absolute shift of the center frequency of the spectral profile. In the following sections the determination of each of these quantities will be discussed in more detail.

\section{DENSITY MEASUREMENTS}

More important than being truly nonintrusive, a diagnostic method should give representative and reproducible results. In a general pulsed LIF experiment, the observed fluorescence light intensity will be proportional to the lasercreated upper state density after the laser pulse, which in turn will be proportional to the initial lower state density before the laser pulse. The pulse-integrated fluorescence signal $S_{f}$ will therefore be proportional to the density $N_{i}$ of particles in this lower state $i$, and the LIF technique can effectively probe this density. These general characteristics of the relation between $S_{f}$ and $N_{i}$ can be expressed in the following way: ${ }^{37}$

$$
S_{f}=N_{i} \cdot \eta_{\mathrm{exc}} \cdot q_{f} \cdot \eta_{\mathrm{det}} \cdot
$$

In this equation the detection efficiency $\eta_{\text {det }}$ is the signal generated per fluorescence photon. This factor is only determined by the characteristics of the experimental setup, and its value can be estimated from the solid angle that is captured by the detection optics, the transmission of these optics, the quantum efficiency and gain of the photon detector, etc.

The fluorescence quantum yield $q_{f}$ is given by the Stern-Vollmer factor: 


$$
q_{f}=\frac{A_{k l}}{A_{k}+Q}=A_{k l} \cdot \tau_{k}
$$

This factor is the ratio between the spontaneous emission rate $A_{k l}$ of level $k$ on the transition to level $l$ where the fluorescence is monitored, and the total depopulation rate of the excited state, which is the sum of the total spontaneous emission rate $A_{k}$ and the nonradiative depopulation (quenching) rate $Q$ of level $k$. The total depopulation rate determines the lifetime $\tau_{k}$ of this state. The excitation efficiency $\eta_{\text {exc }}$ generally contains the excitation cross section and the laser photon fluence (derived from the laser beam intensity), and it depends on the temporal and spectral profile of the laser pulse as well as on the (existence of) couplings of the excited upper state with other states. ${ }^{38,39}$ The derivation of an analytical expression for $\eta_{\text {exc }}$ involves the solution of a set of coupled rate equations for the population of all the levels involved. The value of the proportionality constants can alternatively and more reliably be obtained from a calibration. This is the usual approach to get quantitative density information from a LIF experiment. The calibration procedure for the LIF detection of ground-state hydrogen atoms is the subject of Sec. VII.

The linear correspondence (1) between the LIF signal and the probed lower state density holds not only in the so-called linear limit, i.e., for low laser power and low particle density, but also for higher laser power (as is quite common in two-photon excitation LIF experiments), as long as the excitation laser conditions do not change. The danger of the LIF technique is in the Stern-Vollmer factor. When uncontrolled depopulation pathways for the laser-excited upper state exist, the value of this factor will change and the observed signal will no longer be proportional to the density of the probed state. This is a serious threat to the diagnostic value of the method.

The most common uncontrolled depopulation mechanism is the collisional quenching of the upper state, which occurs when numerous collisions take place during the radiative lifetime of the excited state, i.e., $Q>A_{k}$. This is a wellknown problem in the application of LIF in experiments at a high density, as, for instance, encountered in combustion research, but in the field of (low-temperature) plasma research, where the quenching by electrons can be very efficient, the importance of fluorescence quenching is easily underestimated. As already addressed in Sec. III, the mere application of an intense UV laser for two-photon LIF experiments in plasmas can create a significant (extra) electron density which potentially leads to a "self-quenching" of the fluorescence. ${ }^{34}$

The occurrence of quenching can be readily checked in a time-resolved LIF experiment, where the effective lifetime $\tau_{k}$ of the upper state $k$ is measured. This lifetime is directly related to the quenching as can be seen from Eq. (2). In fact, a measurement of the lifetime will establish the value of the Stern-Vollmer factor, which potentially allows one to correct the density values accordingly. Time-resolved measurements of the fluorescence decay of $\mathrm{H}$ in the $n=3$ state require a special fast PMT and a careful deconvolution of the recorded signal with the response function of the detection system to be able to determine the decay time with sufficient accuracy. These time-resolved LIF measurements on $\mathrm{H}$ in the expanding thermal $\mathrm{Ar}-\mathrm{H}$ plasma are reported in detail and compared with model calculations in the above mentioned paper. ${ }^{34}$ From that study we conclude that quenching of the $\mathrm{H}$ fluorescence from the ETP does not occur. A decay time of $(10.0 \pm 0.5) \mathrm{ns}$ has been observed over the entire range of plasma parameters encountered in the $\mathrm{Ar}-\mathrm{H}$ plasma jet. Summarizing the discussion in Ref. 34, this is exactly the decay time expected in the case of a statistical distribution of the population over the $n=3$ sublevels. Arguments that the $10 \mathrm{~ns}$ decay time is the result of quenching of the $15.5 \mathrm{~ns}$ $3 d \rightarrow 2 p$ natural decay time can be discarded, since the same decay time has been found for a large range of ground-state $\mathrm{H}$ and electron densities. Notice that a pressure-dependent measurement, normally performed to generate a SternVollmer plot in a collisional quenching study, is in this case experimentally very difficult, since a change in background pressure will affect the characteristics of the expanding plasma jet all together.

Another uncontrolled depopulation mechanism that might occur is the amplified spontaneous emission (ASE) of the laser-excited state. The ASE will propagate within a small solid angle around the laser beam axis, and consequently, the upper state population lost in this way will not be observed by the LIF detection branch. ASE of Balmer- $\alpha$ radiation $\left(\mathrm{H}_{\alpha}, 656 \mathrm{~nm}\right)$ in a two-photon LIF experiment for detection of ground-state $\mathrm{H}$ has been reported both for measurements in a flame, ${ }^{40}$ as well as for measurements in a glow discharge plasma. ${ }^{20}$ In these studies the possibility of explicitly using the ASE signal for the detection of $\mathrm{H}$ is investigated. In the experiments on the ETP reported here, no ASE of $\mathrm{H}_{\alpha}$ light has been observed.

A further phenomenon that can disturb the proportionality between lower-state density and an observed LIF signal is the production of the measured atoms by the UV laser itself via photodissociation of molecules. In that way, the laser generates its own signal. This is commonly recognized and described in a number of papers. ${ }^{16,21,23}$ The effect can even be turned into an advantage by using it to identify an impurity and measure its density via a careful analysis of the line profile in the excitation spectrum ${ }^{16,21}$ (see also Sec. V). In the expanding thermal plasma generated from a mixture of $\mathrm{Ar}$ and $\mathrm{H}_{2}$, the only species that could produce $\mathrm{H}$ atoms from photodissociation is molecular hydrogen. We checked the possibility of $205 \mathrm{~nm}$ photodissociation of $\mathrm{H}_{2}$ by filling the vessel with pure molecular hydrogen at room temperature. For the highest laser intensity used in our experiments, we did not observe any H LIF signals for pressures up to $100 \mathrm{~Pa}$. This does not fully exclude that photodissociation of $\mathrm{H}_{2}$ occurs in the plasma, as was also pointed out by Miyazaki et al. ${ }^{23}$ While the effect is negligible for room-temperature $\mathrm{H}_{2}$, it could still be noticable for hot molecules when the photodissociation cross section is sufficiently enhanced for vibrationally excited molecules. The relative importance of the contribution of photodissociation to the H LIF signal depends in that case among others on the amount of $\mathrm{H}_{2}$ present in the plasma jet.

Probably the best way to check for photodissociation is 


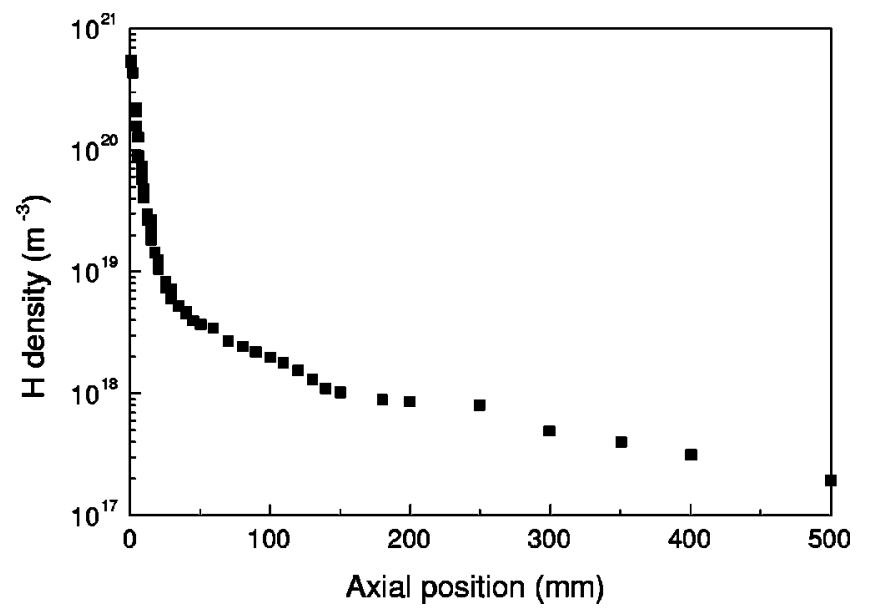

FIG. 5. Atomic hydrogen density in the Ar-H plasma jet as a function of the distance from the expansion nozzle. The axial density profile is derived from spectral scans taken with the laser beam perpendicular to the expansion (z) axis (perpendicular configuration in Fig. 1). The density scale is calibrated via a titration of $\mathrm{H}$ generated in a flow-tube reactor, as described in Sec. VII A.

to measure the laser-intensity dependence of the two-photon LIF signal from the plasma jet. From Fig. 3 we have already seen that the H LIF signal shows a quadratic dependence on the laser intensity over the entire range of laser pulse energies used in our measurements. When photodissociation by the UV laser occurs, a higher order dependence should be observed, since one extra photon is needed to first generate the atom to be detected. ${ }^{16}$ We therefore conclude that in this plasma there is no contribution to the H LIF signal from photodissociation by the UV laser.

Figure 5 gives the atomic hydrogen density in the expanding $\mathrm{Ar}-\mathrm{H}$ plasma as a function of the distance $z$ from the nozzle, i.e., along the symmetry axis of the plasma jet $(r=0)$. The measurements have been performed with the laser perpendicular to the plasma jet axis. Notice that the density profile (i.e., its shape) is determined from the LIF measurements as described above, while the absolute scale for the densities is obtained from a separate calibration as described in Sec. VII A. The H density on the expansion axis starts at $\approx 5 \times 10^{20} \mathrm{~m}^{-3}$ at $1 \mathrm{~mm}$ behind the nozzle and drops more than two orders of magnitude in the supersonic part of the expansion $(0<z \leqq 20 \mathrm{~mm})$. It then further decays at a slower rate in the region of the shock front $(20 \mathrm{~mm} \leqslant z$ $\$ 100 \mathrm{~mm})$ and in the subsonic part of the expansion $(z$ $\gtrsim 100 \mathrm{~mm}$ ). This axial density profile was quite a surprise to us since it seems to deny the existence of a stationary shock wave structure in the expansion. All the peculiar expansion properties of the $\mathrm{Ar}-\mathrm{H}$ plasma jet are discussed in a separate paper. $^{32}$

\section{TEMPERATURE MEASUREMENTS}

The local atom temperature is derived from the width of the spectral profile of the two-photon transition. The experimentally observed linewidth is the result of the convolution of all contributions to the width. The main contribution comes from the Doppler broadening that results from the velocity distribution of the ground-state atoms. The general expression for the Doppler width $\Delta \nu_{D}$ (FWHM) of a twophoton transition, induced by the absorption of one photon with frequency $\nu_{1}$ and one photon with frequency $\nu_{2}$ with an angle $\theta$ between their $k$ vectors, is ${ }^{39}$

$$
\Delta \nu_{D}=2\left(\nu_{1}^{2}+\nu_{2}^{2}+2 \nu_{1} \nu_{2} \cos \theta\right)^{1 / 2} \cdot \bar{v} / c,
$$

where $\bar{v}$ is the average velocity of the atom or molecule and $c$ is the speed of light. In the case of absorption of two photons from one single laser beam with a frequency of half the transition frequency $\nu_{0}\left(\nu_{1}=\nu_{2}=\nu_{0} / 2, \theta=0\right)$, and with a Maxwellian velocity distribution $(\bar{v}=\sqrt{2 \ln 2 \cdot k T / m})$, this expression reduces to the well-known relation between the Doppler width and the temperature $T$ :

$$
\Delta \nu_{D}=\nu_{0} / c \cdot \sqrt{8 \ln 2 \cdot k T / m}=7.16 \times 10^{-7} \nu_{0} \cdot \sqrt{T / M},
$$

with $k$ the Boltzmann constant, $m$ the mass of the atom or molecule, and $M$ its weight in amu. The Doppler width $\Delta \nu_{D}$ is convoluted twice with the linewidth $\Delta \nu_{L}\left(\approx 0.2 \mathrm{~cm}^{-1}\right)$ of the UV laser radiation. Assuming a Gaussian spectral profile for the latter, the experimentally observed linewidth $\Delta \nu$ is simply given by $\Delta \nu=\sqrt{\Delta \nu_{D}^{2}+2 \Delta \nu_{L}^{2}}$. In the case of atomic hydrogen, the error in $\Delta \nu_{D}$, and thus in the temperature, introduced by this squared summation when the laser spectral profile is non-Gaussian, is expected to be small since the observed linewidths are typically one order of magnitude larger than the laser linewidth.

When the above expressions are applied for the determination of the kinetic temperature from the observed linewidth, one has to ascertain that there are no other effects that have a significant contribution to this width. One effect that can be safely neglected is saturation broadening. The degree of saturation of a transition is given by the saturation parameter, which is basically the ratio between the induced and the spontaneous emission rate of the excited level. ${ }^{37}$ Since the induced emission rate is directly proportional to the excitation cross section, the saturation parameter for a two-photon transition will generally be small. More direct evidence that we can neglect saturation broadening in our $\mathrm{H}$ measurements comes of course from Fig. 3, which shows that the $\mathrm{H}$ twophoton excitation remains in the quadratic regime under the present experimental conditions.

Another effect that should be seriously considered when determining temperatures from LIF measurements in plasmas is Stark broadening. In the plasma investigated in this work it will not significantly contribute to the experimentally observed linewidth, since the Stark broadening of the $3 \leftarrow 1$ transition in atomic hydrogen, which is mainly determined by the Stark coefficients of the $n=3$ level, is negligible for the range of electron densities and temperatures encountered in the expanding thermal $\mathrm{Ar}-\mathrm{H}$ plasma $\left(n_{e} \leqslant 10^{19} \mathrm{~m}^{-3}, T_{e}\right.$ $\approx 0.3 \mathrm{eV}){ }^{41}$

There is, however, one effect that could seriously disturb the temperature determination, and that is the photodissociation of molecules by the UV laser by which atoms are produced that are subsequently measured in the same laser pulse. This effect is already mentioned in the density section (IV) since it also affects the proportionality between the initially present lower-state density and the observed LIF signal. The UV photon energy $(\approx 6 \mathrm{eV})$ is sufficient to dissoci- 


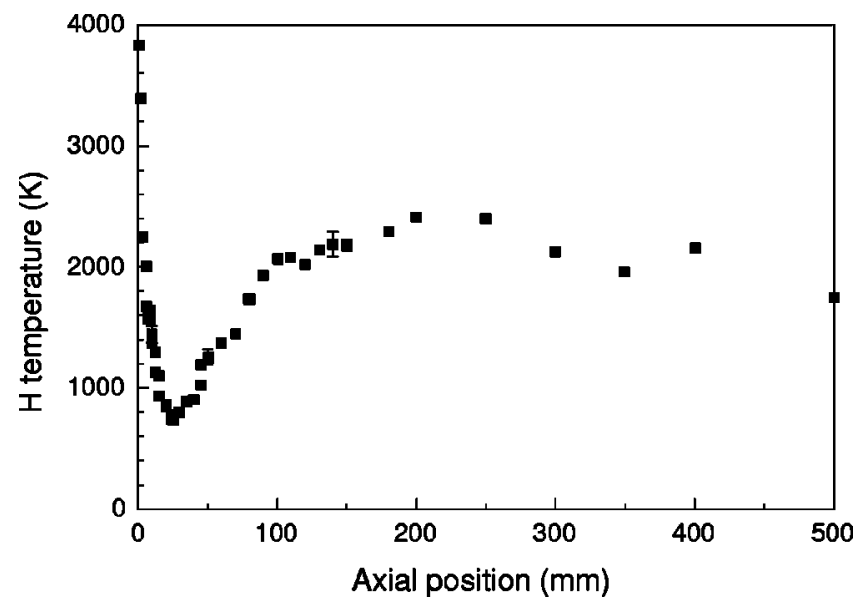

FIG. 6. Axial temperature profile of atomic hydrogen in the expanding $\mathrm{Ar}-\mathrm{H}$ plasma. The temperature data are derived from the same spectral scans as the density data in Fig. 5.

ate a large variety of molecules. Depending on the excess energy, the atomic fragments end up with more than their thermal share of kinetic energy. This gives rise to a large Doppler width and an apparent high atom temperature. Observations of high atomic hydrogen temperatures in rf, microwave, and dc discharges have been associated with photolysis of $\mathrm{NH}_{3}, \mathrm{H}_{2} \mathrm{~S}, \mathrm{C}_{2} \mathrm{H}_{2}, \mathrm{C}_{2} \mathrm{H}_{4}$, and $\mathrm{Si}_{2} \mathrm{H}_{6} \cdot{ }^{16,21,23}$ In fact, "the photolytically produced atoms bear a spectral profile signature characteristic of their precursor molecule," and the Doppler width can be used to determine the excess translational energy enabling an identification of the precursor molecule. ${ }^{16}$ As already pointed out in Sec. IV, the contribution of photolytically produced atoms to the H LIF signal from the expanding $\mathrm{Ar}-\mathrm{H}$ plasma is negligible. Special care, however, is needed in the case of H LIF measurements taken from the flow-tube reactor that is used for the calibration of the H LIF signals (see Sec. VII A). The flow-tube reactor uses a remote microwave plasma to produce $\mathrm{H}$ atoms, and these plasmas are prone to produce ammonia with only small quantities of $\mathrm{N}_{2}$ present in the plasma. ${ }^{16}$

The atomic hydrogen temperature profile along the expansion axis of the Ar-H plasma jet is presented in Fig. 6 . The temperature data in this figure have been derived from the same spectral scans as the density data in Fig. 5. The accuracy in the temperature determination depends on the signal-to-noise $(\mathrm{S} / \mathrm{N})$ ratio of the LIF signal, and on the ratio between the Doppler width of the transition and the linewidth of the excitation laser. Due to the small $\mathrm{H}$ mass, the current linewidth of the UV laser radiation is never the accuracy limiting factor in the $\mathrm{H}$ temperature determination, not even at room temperature. The profile clearly shows the rapid cooling of the plasma from $\approx 4000 \mathrm{~K}$ to $<800 \mathrm{~K}$ in the supersonic part of the expansion, and the subsequent temperature rise over the shock region of the expansion due to the conversion of directed kinetic energy into random kinetic energy when the plasma particles collide with the background gas. In contrast to the density profile in Fig. 5, the temperature profile does exhibit a stationary shock wave structure, thereby constituting a scientific puzzle that is further discussed in Refs. 32 and 35.

\section{VELOCITY MEASUREMENTS}

Besides determining the velocity spread, and from that the temperature, of the particles in the plasma jet from the Doppler width, the Doppler shift of the center frequency of the transition can be used to determine the average velocity of these particles. The Doppler shift can be determined either in a relative or in an absolute way. To determine the Doppler shift in a relative way, a Doppler-shifted signal must be compared to a nonshifted signal, which usually requires two laser beams. An absolute determination of the Doppler-shift "only" requires an absolute frequency scale, and then the shift is obtained from a comparison of the center frequency with the literature value, but obviously in that case the laser frequency needs to be accurately calibrated. In our case, this is accomplished by using a part of the red dye-laser output for the simultaneous recording of the absorption spectrum of molecular iodine, as is described in Sec. II.

An example of such a simultaneous recording is given in Fig. 4. In the region of the fundamental dye-laser frequency, the $I_{2}$ spectrum shows many absorption lines that are tabulated in frequency to an accuracy of $0.015 \mathrm{~cm}^{-1} .33$ The width of the $\mathrm{I}_{2}$ absorption lines, which is at room temperature mainly determined by the underlying hyperfine structure, amounts to $\approx 0.03 \mathrm{~cm}^{-1}$. The medium is therefore very well suited for the frequency calibration of pulsed dye lasers whose bandwidth is usually in the same order of magnitude (in our case $0.07 \mathrm{~cm}^{-1}$ ), and the method is widely used in spectroscopic applications.

Since the Doppler-shift method determines velocities relative to the velocity of light $\left(\delta \nu / \nu_{0}=v / c\right)$, accurate results are not to be expected a priori, and it is worthwhile to address the topic of accuracy into somewhat more detail. In the applied method, the error in the velocity has several sources. The error in the calibrated frequency scale depends on the stepsize in the spectral scan, the signal-to-noise $(\mathrm{S} / \mathrm{N})$ ratio in the $\mathrm{I}_{2}$ absorption spectrum, and is ultimately limited by the bandwidth of the laser. The attainable minimum error in the (fundamental) frequency scale is $\approx 0.01 \mathrm{~cm}^{-1}$. A further error comes from the determination of the center position of the two-photon transition from the Gaussian fit to the $\mathrm{H} \mathrm{LIF}$ signal. The error in the observed center frequency depends again on the stepsize in the spectral scan and on the $\mathrm{S} / \mathrm{N}$ ratio of the H LIF signal. In practice, the overall error in the velocity is mainly determined by this contribution.

A last uncertainty concerns the literature value for the transition frequency. Accurate values for the energies of the individual $n=3$ sublevels can, for instance, be found in Ref. 42 , but since the maximum energy separation between these levels is smaller than the bandwidth of the $205 \mathrm{~nm}$ laser radiation (and much smaller than the Doppler width), these levels are not resolved and the resulting center frequency will be at an averaged value. This leaves some room for the exact reference value to take for the velocity determination. A reference transition frequency could, for instance, be obtained by taking an average that accounts for the selection rules and the relative excitation cross sections of the twophoton transition. The reference value used in this work, however, is obtained from the experiment itself. In a mea- 


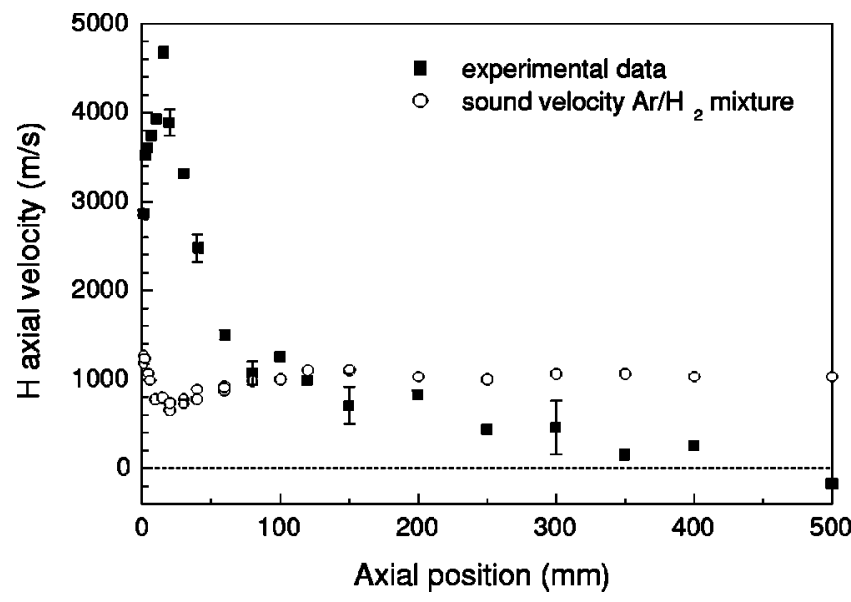

FIG. 7. Axial component of the atomic hydrogen velocity in the $\mathrm{Ar}-\mathrm{H}$ plasma jet as a function of the distance from the nozzle. The velocity is determined from the Doppler shift in the spectral scans that are taken with the laser beam counterpropagating with respect to the expansion (antiparallel configuration in Fig. 1). The laser frequency is calibrated by simultaneously recording the $I_{2}$ absorption spectrum. The local acoustic velocity of the $\mathrm{Ar}-\mathrm{H}$ mixture that is derived from the temperature data in Fig. 6 is plotted as a reference.

surement of the radial velocity in a radial cross section through the ETP, symmetry arguments are used to put the radial velocity on the symmetry axis of the expansion to zero. This leads to the value $\nu_{0}=97492.315 \mathrm{~cm}^{-1}$. This value is very close to the averaged literature value that accounts for a pure statistical distribution of the population over all the $n=3$ sublevels, which is consistent with the observed lifetime of the excited state, ${ }^{34}$ as is discussed in Sec. IV.

The resulting maximum accuracy that can be attained in the determination of the velocity of atomic hydrogen from the Doppler shift of the transition frequency in the twophoton LIF measurements in the Ar-H plasma jet is $\approx 200$ $\mathrm{m} / \mathrm{s}$, which is a fraction of less than $10^{-6}$ of the speed of light. This value is consistent with the $\mathrm{S} / \mathrm{N}$ ratio that we experimentally observe in our velocity measurements. This accuracy is nearly laser linewidth limited and is certainly sufficient to gain valuable information about the flow pattern in the expanding plasma jet, where $\mathrm{H}$ atom velocities in the range 0 to $\approx 4500 \mathrm{~m} / \mathrm{s}$ are observed.

Figure 7 shows a measurement of the atomic hydrogen axial velocity component in the expanding $\mathrm{Ar}-\mathrm{H}$ plasma as a function of the distance $z$ from the nozzle. Notice that these measurements require a laser beam that is (anti-)parallel to the expansion axis, i.e., counterpropagating with respect to the expansion (see Sec. II). As a reference, we plotted in the same figure the local acoustic velocity of the $\mathrm{Ar}-\mathrm{H}$ mixture as it has been determined from the $\mathrm{H}$ temperature data from Fig. 6 using simple gas-dynamic formulas. The $\mathrm{H}$ axial velocity profile clearly shows the last part of the rapid supersonic acceleration in the expansion region up to a maximum velocity of $\approx 4200 \mathrm{~m} / \mathrm{s}$, and the subsequent deceleration in the shock region due to the increasing interaction with the background gas, until the velocity becomes subsonic again at $z \approx 100 \mathrm{~mm}$. From the combined density (Fig. 5) and velocity (Fig. 7) data it is concluded that the forward flux of $\mathrm{H}$ is not conserved over the shock region in the expansion of a thermal Ar-H plasma, which is a clear deviation of "normal" supersonic expansion behavior as described by supersonic expansion theory. ${ }^{43-45}$ The in-depth physical discussion of the expansion properties of the plasma jet is left to a separate paper. $^{32,35}$

\section{DENSITY CALIBRATION}

The density information gained in a LIF experiment, although in principal quantifiable, is in practice qualitative in nature. It was already mentioned above that, in order to get quantitative results from a LIF experiment, the setup usually needs to be calibrated. In the case of short-lived species like radicals or ions, calibration is not trivial. In the Introduction, three different calibration approaches have been mentioned. Here, two of these approaches are used to calibrate the setup for the atomic hydrogen measurements. The first approach uses a "flow-tube reactor" as a source of ground-state $\mathrm{H}$ atoms, in which the atomic hydrogen concentration is determined by a titration. This method is based on wellestablished flow reactor and titration techniques in chemistry, ${ }^{24}$ and is commonly accepted for LIF measurements of ground-state atomic radical densities in plasmas. The method, though, is experimentally quite elaborate and needs careful execution. Of course, one needs to have available a flow-tube reactor specifically adapted to the plasma chamber in which the LIF measurements are performed, and one should attain proper control of the chemistry in this reactor for a reliable calibration result.

The second approach avoids the cumbersome generation of a known amount of atomic hydrogen radicals from an independent source. Instead, a LIF signal is generated from a fixed pressure of a different, stable species by a comparable two-photon excitation scheme. The $\mathrm{H}$ density in the plasma jet can then be determined from the ratio of the LIF signals when the ratio of the two-photon excitation cross sections is known. This method is more elegant and experimentally far more easy than the first one, but it relies on critical cross section data not yet confirmed by other experiments.

We will first address both methods separately, and make an assessment based on their comparison afterwards.

\section{A. Titration in a flow-tube reactor}

A scheme of the flow-tube reactor is depicted in Fig. 8. It has been designed to allow implementation in the setup of Fig. 1 without changing any of the other components. A small amount of molecular hydrogen that is diluted in helium flows through a quartz tube that is mounted through the center of a Beenakker-type microwave cavity. ${ }^{46}$ Atomic hydrogen is produced in the flow-tube by dissociation of $\mathrm{H}_{2}$ in the microwave discharge (typical power of $50 \mathrm{~W}$ ) that is sustained in the cavity. The main flow continues down the quartz tube and a $0.5 \mathrm{~m}$ long teflon tube, transporting $\mathrm{H}$ atoms to a position $60 \mathrm{~cm}$ downstream from the discharge and only 1 or $2 \mathrm{~mm}$ away from the scattering volume of the laser where they are detected with identical excitation and detection geometry and efficiency as in the ETP. The tube material has been chosen to minimize the $\mathrm{H}$ atom loss be- 


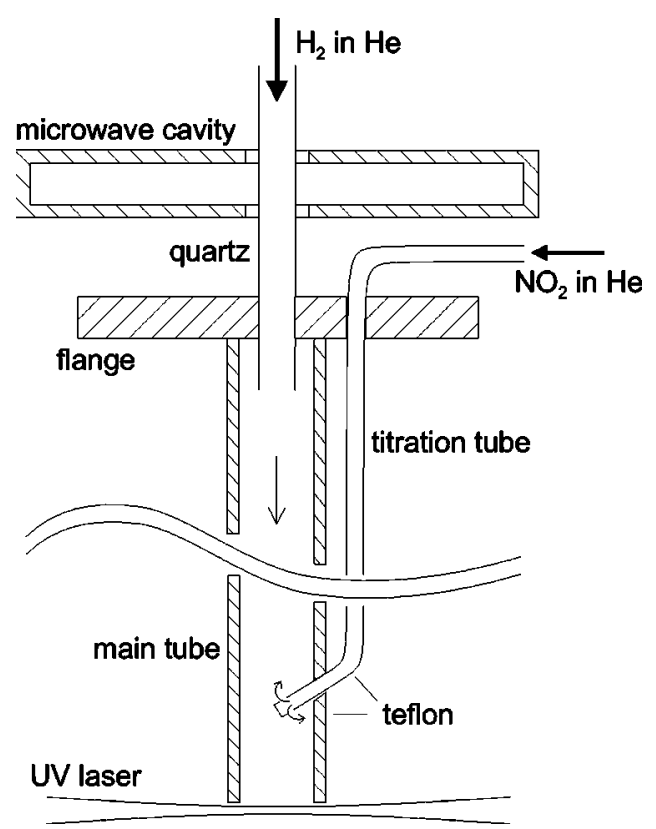

FIG. 8. Scheme of the flow-tube reactor that is used for the calibration of the atomic hydrogen fluorescence signal. $\mathrm{H}$ is produced from a $\mathrm{He}-\mathrm{H}_{2}$ flow in a (Beenakker-type) microwave cavity and transported via inert quartz and teflon tubes to the laser excitation volume. The original amount of $\mathrm{H}$ present in this volume is determined via a titration with an accurately controlled $\mathrm{NO}_{2}$ flow that can be admixed to the main flow just before the excitation volume.

tween the microwave discharge and the point where $\mathrm{H}$ is detected. The length of the tube, or better the transport time, ensures that no excited $\mathrm{H}$ atoms arrive at the scattering volume of the laser.

The amount of $\mathrm{H}$ present in the scattering volume and responsible for the observed signal is determined via a titration with $\mathrm{NO}_{2}$. The $\mathrm{NO}_{2}$ comes from a calibrated mixture of $2.11 \% \mathrm{NO}_{2}$ in $\mathrm{He}$ that can be added to the main flow via a small insert that enters the main transport tube near the end of it. The end of the small titration tube is perforated around its perimeter to enhance the mixing with the main flow. The titration actually determines the $\mathrm{H}$ density in the mixing region. To ensure that this density is as close as possible to the $\mathrm{H}$ density responsible for the LIF signal in the laser scattering volume, the mixing region should be as close to the exit of the main tube as possible. The $3.5 \mathrm{~cm}$ distance between the end of the titration tube and the exit of the main tube, however, is the minimum distance needed for the titration reaction to be complete, as will be discussed below. This distance is small compared to the length of the main tube, and it can therefore be safely assumed that the ground-state $\mathrm{H}$ density is constant over the last $3.5 \mathrm{~cm}$ of the flow tube. All flows in the flow-tube reactor are accurately controlled with mass flow controllers.

Upon addition of $\mathrm{NO}_{2}, \mathrm{H}$ atoms are lost in the fast reaction:

$$
\mathrm{H}+\mathrm{NO}_{2} \rightarrow \mathrm{OH}+\mathrm{NO},
$$

with a rate constant $k$ of $1.3 \times 10^{-10} \mathrm{~cm}^{3} / \mathrm{s}^{47}$ Solving the symmetric set of rate equations

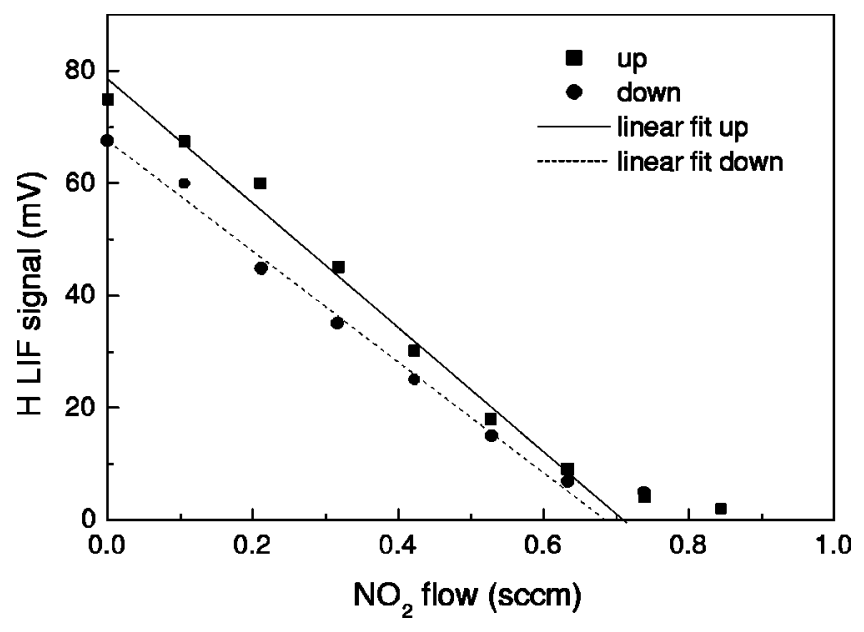

FIG. 9. $\mathrm{H}$ fluorescence signal from the flow-tube reactor vs the admixed $\mathrm{NO}_{2}$ flow. The dependence is recorded both in a series with increasing flow (up) and in one with decreasing flow (down). The linear fits to these series both intersect the ordinate at a value for the $\mathrm{NO}_{2}$ flow of $(0.69 \pm 0.04) \mathrm{sccm}$.

$$
\frac{d n_{\mathrm{H}}}{d t}=-k n_{\mathrm{H}} n_{\mathrm{NO}_{2}}=\frac{d n_{\mathrm{NO}_{2}}}{d t}
$$

for the densities $n_{\mathrm{H}}$ and $n_{\mathrm{NO}_{2}}$ of $\mathrm{H}$ and $\mathrm{NO}_{2}$, respectively, shows that when $t \rightarrow \infty$, i.e., when the reaction is complete, the remaining $\mathrm{H}$ density depends linearly on the initial $\mathrm{NO}_{2}$ density. Figure 9 shows a typical titration curve, i.e., a measurement of the $\mathrm{H}$ fluorescence signal from the flow-tube reactor as a function of the admixed $\mathrm{NO}_{2}$ flow. The dependence is recorded both in a series with increasing flow (up) and in one with decreasing flow (down). The H LIF signal decreases linearly with increasing $\mathrm{NO}_{2}$ flow, as it should, until it becomes indistinguishable from the background (end point of titration). Given the 1:1 stoichiometric ratio of the titration reaction, the $\mathrm{NO}_{2}$ density $n_{\mathrm{NO}_{2}}^{(T)}$ at the $x$ intercept in Fig. 9, which is necessary to remove all $\mathrm{H}$ atoms, equals the original $\mathrm{H}$ density $n_{\mathrm{H}}$ at the beginning of the titration that gives rise to the LIF signal at the $y$ intercept, or

$$
n_{\mathrm{H}}=n_{\mathrm{NO}_{2}}^{(T)}=n_{\text {tot }} \cdot \frac{\Phi_{\mathrm{NO}_{2}}^{(T)}}{\Phi_{\text {tot }}}=\frac{p}{k T} \cdot \frac{\Phi_{\mathrm{NO}_{2}}^{(T)}}{\Phi_{\text {tot }}},
$$

with $p$ and $T$ the pressure and temperature in the vessel, respectively, $k$ Boltzmann's constant, $\Phi_{\mathrm{NO}_{2}}^{(T)}$ the $\mathrm{NO}_{2}$ flow rate, and the subscript "tot" denoting the total density or flow. During the titration, the total flow $\Phi_{\text {tot }}$ is kept constant in order to keep the pressure $p$ in the vessel constant. The increasing flow of the calibrated $\mathrm{NO}_{2}-\mathrm{He}$ mixture is balanced by a decreasing additional pure $\mathrm{He}$ flow in the small titration tube.

The first equality in Eq. (7) only holds under certain conditions. First of all, the titration reaction should be complete, i.e., there should be enough time for all the particles to react before they reach the laser scattering volume. This condition can be quantified by evaluating the solution of the set of equations (6) for the case that the initial $\mathrm{NO}_{2}$ density $n_{\mathrm{NO}_{2}, 0}($ at $t=0)$ approaches the initial $\mathrm{H}$ density $n_{\mathrm{H}, 0}$, i.e., near the end point of titration. This yields 


$$
n_{\mathrm{H}}(t)=\frac{n_{\mathrm{H}, 0}}{1+k n_{\mathrm{H}, 0} t} .
$$

The end point of titration $\left(n_{\mathrm{NO}_{2}, 0}=n_{\mathrm{H}, 0}\right)$ is only sharply determined $\left[n_{\mathrm{H}}(t)=0\right]$ if $k n_{\mathrm{H}, 0} t \gg 1$. This is the condition for linearity and complete titration.

In addition, the $\left[\mathrm{NO}_{2}\right] /[\mathrm{H}]$ stoichiometry should be $1: 1$. Other reactions that could change the stoichiometry should only play a minor role. The secondary reactions $\mathrm{OH}+\mathrm{OH} \rightarrow \mathrm{H}_{2} \mathrm{O}+\mathrm{O}$ and $\mathrm{O}+\mathrm{OH} \rightarrow \mathrm{O}_{2}+\mathrm{H}$ are significantly reduced at the low $\mathrm{H}$ concentrations in the flow-tube reactor, ${ }^{17,48}$ and other interfering reactions such as $\mathrm{O}+\mathrm{NO}_{2} \rightarrow \mathrm{O}_{2}+\mathrm{NO}$ and $\mathrm{OH}+\mathrm{H}_{2} \rightarrow \mathrm{H}_{2} \mathrm{O}+\mathrm{H}$ are made negligible by diluting both $\mathrm{NO}_{2}$ and $\mathrm{H}_{2}$ in He. ${ }^{17}$ The second equality in Eq. (7) implies the assumption of efficient mixing between the $\mathrm{NO}_{2} / \mathrm{He}$ flow from the titration tube and the main flow.

A proper density calibration furthermore avoids a "selfgenerated signal." As already stated in Sec. IV, the photolysis of $\mathrm{H}$ containing molecules by the UV laser, producing $\mathrm{H}$ atoms that are subsequently measured in the same laser pulse, disturbs the proportionality between the initially present lower-state density and the observed LIF signal. This effect is likely to occur in the flow-tube reactor since it uses a microwave plasma for the production of $\mathrm{H}$ atoms, and these plasmas are known to produce, for instance, ammonia with only small quantities of nitrogen present in the plasma. ${ }^{16}$ The occurrence of UV photolysis of impurities from the flow-tube reactor can be easily checked by examining the Doppler width of the H LIF signal. This should yield a temperature close to room temperature. As already pointed out in Sec. V, impurities can be identified by an apparent high atom temperature. The Doppler width of the $\mathrm{H}$ LIF signal from the flow-tube reactor corresponded to a temperature of $\approx 400 \mathrm{~K}$, which is slightly elevated above room temperature. We strongly believe, however, that this is not the result of photolysis of impurities. It is more likely the residual temperature from the microwave plasma in which the $\mathrm{H}$ atoms are produced.

In the titration shown in Fig. 9, the end point of titration is not very sharply determined and is therefore derived from a linear fit to all the data points except for the ones at the two highest $\mathrm{NO}_{2}$ flow rates, yielding $\Phi_{\mathrm{NO}_{2}}^{(T)}=0.69 \pm 0.04 \mathrm{sccm}$. The corresponding initial $\mathrm{H}$ density can now be derived from Eq. (7) using the settings $p=2.4 \times 10^{2} \mathrm{~Pa}, T=300 \mathrm{~K}$, and $\Phi_{\text {tot }}=504 \mathrm{sccm}$, resulting in $n_{\mathrm{H}}=(7.8 \pm 2.6) \times 10^{19} \mathrm{~m}^{-3}$. The accuracy in $n_{\mathrm{H}}$ is mainly determined by the relatively large uncertainty in the value for the vessel pressure $p$ that was read from a mechanical manometer. This is currently subject to improvement.

We can now evaluate the condition for linearity and complete titration, i.e., the value of $k n_{\mathrm{H}, 0} t$. The reaction time $t$ is determined by the $3.5 \mathrm{~cm}$ distance between the end of the titration tube and the exit of the main tube and by the flow velocity. Using the above values yields $t=2.1 \mathrm{~ms}$ and $k n_{\mathrm{H}, 0} t=21$. This shows that the condition for linearity and complete titration is only just fulfilled. According to Eq. (8), the remaining $\mathrm{H}$ density at the end point of titration is $\approx 5$ $\times 10^{-2} n_{\mathrm{H}, 0}$, which corresponds to a signal value of about 4
$\mathrm{mV}$ in Fig. 9. This is in agreement with the measured signal at the end point of titration that is determined from the linear fit.

The $\mathrm{H}$ fluorescence signal in Fig. 9 is expressed as the amplitude of the PMT signal on the oscilloscope. This signal value sensitively depends on the laser pulse energy $(120 \mu \mathrm{J}$ for this case), the laser frequency, and the degree of overlap of the excitation volume with the detection volume. The difference between the signal value at the beginning of the first measurement series (up) and at the end of the second series (down) is indicative of the reproducibility of the measurement. It is therefore of upmost importance to change as little as possible when switching between the recording of the $\mathrm{H}$ LIF signal from the flow-tube reactor and that from the expanding thermal plasma. In practice this means that the two measurements should be performed directly after each other. Finally, when converting H LIF signal values from the ETP to $\mathrm{H}$ densities one should take into account that the laser linewidth is smaller than the width of the induced transition. Since the $\mathrm{H}$ atom temperature in the two recordings will generally be different, this demands one to compare only spectrally integrated signal values. Thus by scaling the above found $\mathrm{H}$ density with the ratio between the spectrally integrated LIF intensity from the plasma jet and that from the flow-tube reactor, we are able to determine the spatially resolved absolute $\mathrm{H}$ density in the ETP.

Now that we have quantitative $\mathrm{H}$ density values, we can estimate the detection limit of the two-photon excitation laser-induced fluorescence technique for ground-state atomic hydrogen in the plasma jet. From the density measurements we infer a noise-equivalent two-photon LIF detection limit for $\mathrm{H}$ in our experimental setup of $10^{15} \mathrm{~m}^{-3}$. Notice that the solid angle of detection is limited to $4.9 \times 10^{-2} \mathrm{sr}$ by the geometry of our experimental setup. In smaller setups the detection limit can be smaller by a factor proportional to the increase in solid angle. The absolute accuracy of the density measurements depends on the accuracy of the calibration, which is currently limited to $33 \%$. The relative accuracy (reproducibility), however, depends on the signal-to-noise ratio in the LIF measurements and can be orders of magnitude better (see, for instance, Fig. 5).

\section{B. Two-photon excitation of krypton}

The basic idea behind a radical density calibration using a known density of a stable species is to find and use an excitation and detection scheme in a stable species as similar as possible to that of the radical. This warrants a comparable response [Eq. (1)] to the laser excitation, especially when (in addition) the excitation wavelengths are close to each other, which allows the excitation conditions (laser spectral, temporal, and beam profile, intensity, and focusing) to be the same in the two LIF measurements.

The excitation and detection scheme for krypton that is used in the atomic hydrogen density calibration is depicted on the right in Fig. 2. In this scheme $\mathrm{Kr}$ is excited with two $204.2 \mathrm{~nm}$ photons from the $4 p^{6}{ }^{1} S_{0}$ ground state to the $5 p^{\prime}[3 / 2]_{2}$ state. The resulting nonresonant fluorescence from this state to the $5 s^{\prime}[1 / 2]_{1}$ state is detected at $826 \mathrm{~nm}$. Figure 


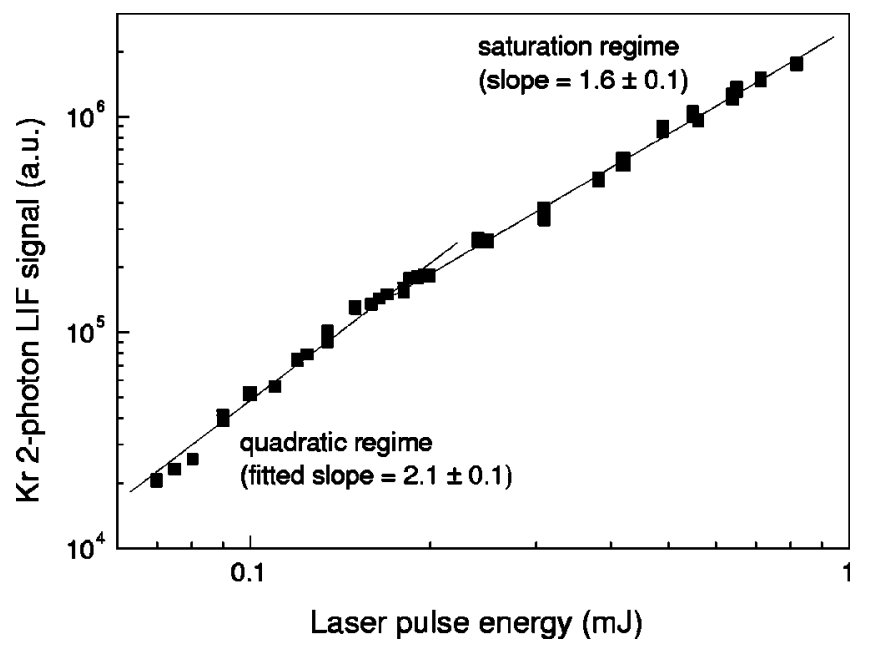

FIG. 10. Two-photon excitation laser-induced fluorescence signal of atomic krypton as a function of the $204 \mathrm{~nm}$ laser pulse energy. The solid lines are fits to the experimental data. Two separate regimes can clearly be distinguished. The Kr LIF signal depends quadratically on the laser intensity for laser pulse energies below $0.18 \mathrm{~mJ}$.

2 clearly shows the resemblance of the $\mathrm{H}$ and $\mathrm{Kr}$ excitation and detection schemes. In both schemes the excitation is on a two-photon transition, which gives a quadratic response to the laser intensity in the small signal limit (low laser intensity). The wavelengths used for excitation are in addition nearly equal and can actually be generated from the same dye solution in the tunable dye laser. This means in practice that we can switch between $\mathrm{H}$ and $\mathrm{Kr}$ measurements within an instant; the laser is tuned to the other wavelength and only the fluorescence filter in front of the PMT has to be replaced. In the quadratic regime and under equal excitation conditions, the ratio of the (temporally and spectrally integrated) fluorescence signals $S_{\mathrm{X}}$ that are given by Eq. (1), where $\mathrm{X}=\mathrm{Kr}$ or $\mathrm{H}$, reduces $\mathrm{to}^{27-29}$

$$
\frac{S_{\mathrm{Kr}}}{S_{\mathrm{H}}}=\frac{n_{\mathrm{Kr}}}{n_{\mathrm{H}}} \frac{\sigma_{\mathrm{Kr}}^{(2)}}{\sigma_{\mathrm{H}}^{(2)}}\left(\frac{I_{\mathrm{Kr}} \nu_{\mathrm{H}}}{\nu_{\mathrm{Kr}} I_{\mathrm{H}}}\right)^{2} \frac{A_{\mathrm{Kr}} \tau_{\mathrm{Kr}}}{A_{\mathrm{H}} \tau_{\mathrm{H}}} \frac{T_{\mathrm{Kr}}}{T_{\mathrm{H}}} \frac{\zeta_{\mathrm{Kr}}}{\zeta_{\mathrm{H}}} .
$$

Here $n_{\mathrm{X}}$ is the ground-state density of $\mathrm{X}$ atoms, $I_{\mathrm{X}}$ is the corresponding laser beam energy, $\nu_{\mathrm{X}}$ is the photon frequency, $A_{\mathrm{X}}$ is the Einstein transition probability for the detected spontaneous emission, $\tau_{X}$ is the lifetime of the excited state, $T_{\mathrm{X}}$ is the transmission of the optical detection branch for the detected fluorescence, and $\zeta_{\mathrm{X}}$ is the detector quantum efficiency at the corresponding wavelength. The solid angle that is captured by the detection optics and the gain of the photon detector are the same in the two LIF measurements. The ratio of the two-photon excitation cross sections $\sigma_{\mathrm{X}}^{(2)}$ has recently been measured to be $\sigma_{\mathrm{Kr}}^{(2)} / \sigma_{\mathrm{H}}^{(2)}=0.62 .^{27}$

For the Kr LIF measurements the vacuum chamber has been filled with $10 \mathrm{~Pa} \mathrm{Kr}$ at room temperature which corresponds to a $\mathrm{Kr}$ density of $2.4 \times 10^{21} \mathrm{~m}^{-3}$. The two-photon transition in $\mathrm{Kr}$, that is induced here for the $\mathrm{H}$ density calibration, is easily saturated. The transition is, for instance, known to readily generate ASE, enhanced by its small Doppler width. ${ }^{49}$ Since Eq. (9) only holds in the quadratic regime, special care has been taken to avoid saturation of the transition. Figure 10 shows a measurement of the Kr fluores-
TABLE I. Values of the parameters involved in the $\mathrm{H}$ density calibration via two-photon LIF of krypton, using Eq. (9). The transmission $T$ of the optical filters in the detection branch for the respective fluorescence wavelengths has been measured with a FT spectrometer. The PMT quantum efficiency $\zeta$ for the $\mathrm{Kr}$ fluorescence wavelength $(826 \mathrm{~nm})$ has been measured relative to the one for the H Balmer- $\alpha$ fluorescence $(656.3 \mathrm{~nm})$ with a ribbon lamp.

\begin{tabular}{lcc}
\hline \hline & $\mathrm{Kr}$ & $\mathrm{H}$ \\
\hline $\mathrm{I}_{\text {laser }}(\mathrm{mJ})$ & 0.12 & 0.39 \\
$\nu\left(\mathrm{cm}^{-1}\right)$ & 48972.985 & 48746.15 \\
$A\left(\mathrm{~s}^{-1}\right)$ & $2.7 \times 10^{7}$ & $4.4 \times 10^{7}$ \\
$\tau(\mathrm{ns})$ & 35.4 & 10.0 \\
$T$ & 0.95 & 0.447 \\
$\zeta$ (relative) & 0.088 & 1.0 \\
\hline \hline
\end{tabular}

cence signal at resonance as a function of the laser pulse energy. Two different regimes can be clearly distinguished. At energies below $0.18 \mathrm{~mJ}$ the $\mathrm{Kr}$ fluorescence yield is a quadratic function of the laser energy. At higher energies the transition starts to saturate and in this regime the $\mathrm{Kr}$ LIF signal has a lower order response to the laser intensity. The $\mathrm{Kr}$ LIF measurements for the $\mathrm{H}$ density calibration have therefore been performed at a $0.12 \mathrm{~mJ}$ laser pulse energy. From Fig. 3 we know that the $\mathrm{H}$ two-photon transition does not saturate for laser pulse energies $\leqslant 1 \mathrm{~mJ}$. For the calibration measurements on $\mathrm{H}$ a $0.4 \mathrm{~mJ}$ laser pulse energy has been used.

As already mentioned in Secs. III and IV, the lifetime $\tau_{\mathrm{H}}$ of the $\mathrm{H} n=3$ excited state has been measured to be $(10.0$ $\pm 0.1) \mathrm{ns}$, which suggests a statistical distribution of the population over the $n=3$ sublevels. This is independent of the position in the plasma jet and of the plasma parameters, as discussed in Ref. 34. The lifetime $\tau_{\mathrm{Kr}}$ of the $\operatorname{Kr} 5 p^{\prime}[3 / 2]_{2}$ state is found to be $(35.4 \pm 2.7) \mathrm{ns}$, in agreement with the value recently measured by Niemi et $a l .{ }^{27}$ This value differs from the previously reported radiative lifetime of this state $\left(26.9 \mathrm{~ns}^{50}\right)$. We obtain the product $A_{\mathrm{Kr}} \tau_{\mathrm{Kr}}$ from the branching ratio of the observed $\mathrm{Kr}$ transition, which is $0.95 .^{50}$ This implies a value of $2.7 \times 10^{7} \mathrm{~s}^{-1}$ for the Einstein coefficient $A_{\mathrm{Kr}}$. Table I summarizes the values of all parameters

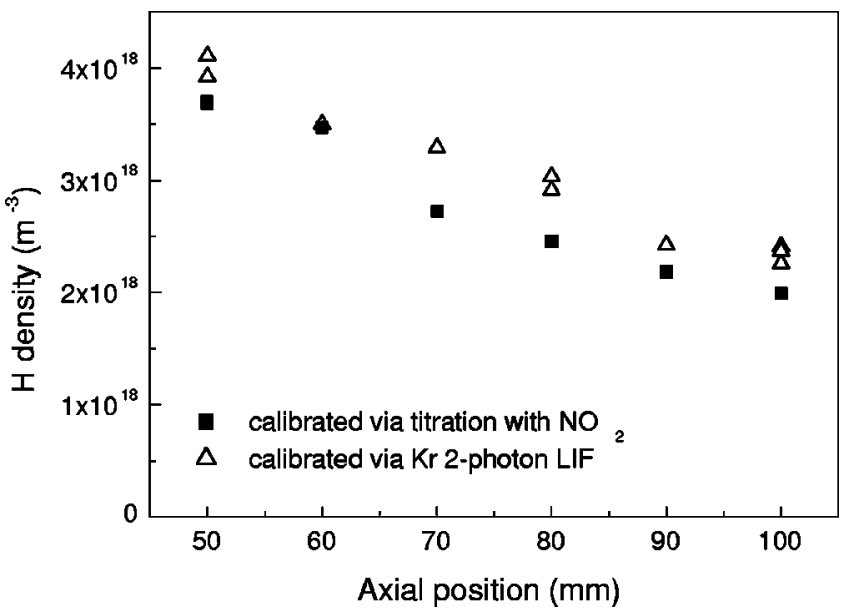

FIG. 11. Comparison between absolute atomic hydrogen density profiles along the expansion axis that are calibrated via a titration in a flow-tube reactor $(\square)$ and via two-photon excitation of krypton $(\triangle)$. 
involved in the $\mathrm{H}$ density calibration via krypton, using Eq. (9).

Figure 11 shows two axial profiles of the absolute atomic hydrogen density in the expansion of a thermal $\mathrm{Ar}-\mathrm{H}$ plasma. One density profile is calibrated via a titration in a flow-tube reactor, the other via a two-photon LIF measurement of krypton. The axial profiles are plotted in the region between 50 and $100 \mathrm{~mm}$ from the cascaded arc nozzle. In this supersonic region the plasma jet characteristics are mainly determined by the cascaded arc geometry and parameter settings, which were the same for the two density profile measurements. The comparison shows that the $\mathrm{H}$ density values determined by the two different methods agree quite well with each other when the two-photon excitation cross-section ratio $\sigma_{\mathrm{Kr}}^{(2)} / \sigma_{\mathrm{H}}^{(2)}=0.62$ from Ref. 27 is used. Actually, there seems to be a small systematic difference between the two profiles. We can alternatively calibrate the new "rare gas method" with the "flow-tube reactor method." Putting the two profiles on top of each other gives $\sigma_{\mathrm{Kr}}^{(2)} / \sigma_{\mathrm{H}}^{(2)}=0.56$. These values agree with each other within the experimental accuracy estimated to be around $50 \%$ in both cases. This comparison therefore validates the $\mathrm{H}$ density calibration via a two-photon LIF measurement of $\mathrm{Kr}$, which is experimentally far more easy than the calibration with the flow-tube reactor once the values of the parameters in Eq. (9) are accurately determined.

\section{ACKNOWLEDGMENTS}

This work is part of the research program of the Dutch Foundation for Fundamental Research on Matter (FOM). It is also financially supported by the Dutch Technology Foundation (STW) and the Euratom foundation. The authors would like to thank Dr. U. Czarnetzki and coworkers at the University of Essen for using their flow-tube reactor, and $\mathrm{H}$. M. M. de Jong, M. J. F. van de Sande, and A. B. M. Hüsken for their skillful technical assistance.

${ }^{1}$ R. F. G. Meulenbroeks, R. A. H. Engeln, M. N. A. Beurskens, R. M. J. Paffen, M. C. M. van de Sanden, J. A. M. van der Mullen, and D. C. Schram, Plasma Sources Sci. Technol. 4, 74 (1995).

${ }^{2}$ J. W. A. M. Gielen, W. M. M. Kessels, M. C. M. van de Sanden, and D. C. Schram, J. Appl. Phys. 82, 2643 (1997).

${ }^{3}$ W. M. M. Kessels, M. C. M. van de Sanden, and D. C. Schram, J. Vac. Sci. Technol. A 18, 2153 (2000).

${ }^{4}$ J. Amorim, G. Baravian, and J. Jolly, J. Phys. D 33, R51 (2000).

${ }^{5}$ J. Bokor, R. R. Freeman, J. C. White, and R. H. Storz, Phys. Rev. A 24, 612 (1981).

${ }^{6}$ W. K. Bischel, B. E. Perry, and D. R. Crosley, Chem. Phys. Lett. 82, 85 (1981).

${ }^{7}$ M. Alden, H. Edner, P. Grafstrom, and S. Svanberg, Opt. Commun. 42, 244 (1982).

${ }^{8}$ L. F. DiMauro, R. A. Gottscho, and T. A. Miller, J. Appl. Phys. 56, 2007 (1984).

${ }^{9}$ M. Heaven, T. A. Miller, R. R. Freeman, J. C. White, and J. Bokor, Chem. Phys. Lett. 86, 458 (1982).

${ }^{10}$ P. Brewer, N. van Veen, and R. Bersohn, Chem. Phys. Lett. 91, 126 (1982).

${ }^{11}$ P. Das, G. Ondrey, N. van Veen, and R. Bersohn, J. Chem. Phys. 79, 724 (1983).

${ }^{12}$ S. G. Hansen, G. Luckman, G. C. Nieman, and S. D. Colson, Appl. Phys. Lett. 51, 898 (1987).
${ }^{13}$ G. C. Herring, M. J. Dyer, L. E. Jusinski, and W. K. Bischel, Opt. Lett. 13, 360 (1988).

${ }^{14}$ B. L. Preppernau, D. A. Dolson, R. A. Gottscho, and T. A. Miller, Plasma Chem. Plasma Process. 9, 157 (1989).

${ }^{15}$ B. L. Preppernau and T. A. Miller, J. Vac. Sci. Technol. A 8, 1673 (1990).

${ }^{16}$ J. R. Dunlop, A. D. Tserepi, B. L. Preppernau, T. M. Cerny, and T. A. Miller, Plasma Chem. Plasma Process. 12, 89 (1992).

${ }^{17}$ A. D. Tserepi, J. R. Dunlop, B. L. Preppernau, and T. A. Miller, J. Appl. Phys. 72, 2638 (1992).

${ }^{18}$ B. L. Preppernau, K. Pearce, A. Tserepi, E. Wurzberg, and T. A. Miller, Chem. Phys. 196, 371 (1995).

${ }^{19}$ U. Czarnetzki, K. Miyazaki, T. Kajiwara, K. Muraoka, M. Maeda, and H. F. Döbele, J. Opt. Soc. Am. B 11, 2155 (1994).

${ }^{20}$ J. Amorim, G. Baravian, M. Touzeau, and J. Jolly, J. Appl. Phys. 76, 1487 (1994).

${ }^{21}$ J. Amorim, G. Baravian, S. Bockel, A. Ricard, and G. Sultan, J. Phys. III 6, 1147 (1996).

${ }^{22}$ J. Larjo, J. Walewski, and R. Hernberg, Appl. Phys. B: Lasers Opt. 72, 455 (2001).

${ }^{23}$ K. Miyazaki, T. Kajiwara, K. Uchino, K. Muraoka, T. Okada, and M. Maeda, J. Vac. Sci. Technol. A 14, 125 (1996).

${ }^{24}$ See, for instance, Reactive Intermediates in the Gas Phase, edited by D. W. Setser (Academic, New York, 1979).

${ }^{25}$ U. Meier, K. Kohse-Hoinghaus, L. Schafer, and C. P. Klages, Appl. Opt. 29, 4993 (1990).

${ }^{26}$ H. F. Döbele, M. Hörl, and M. Röwekamp, Appl. Phys. B: Photophys. Laser Chem. 39, 91 (1986).

${ }^{27}$ K. Niemi, V. Schultz von der Gathen, and H. F. Döbele, J. Phys. D 34, 2330 (2001).

${ }^{28}$ S. Mazouffre, C. Foissac, P. Supiot, R. Engeln, P. J. W. Vankan, D. C. Schram, and N. Sadeghi, Plasma Sources Sci. Technol. 10, 168 (2001).

${ }^{29}$ A. Goehlich, T. Kawetzki, and H. F. Döbele, J. Chem. Phys. 108, 9362 (1998).

${ }^{30}$ J. G. Liebeskind, R. K. Hanson, and M. A. Capelli, Appl. Opt. 32, 6117 (1993).

${ }^{31}$ N. Sadeghi, M. van de Grift, D. Vender, G. M. W. Kroesen, and F. J. de Hoog, Appl. Phys. Lett. 70, 835 (1997).

${ }^{32}$ S. Mazouffre, M. G. H. Boogaarts, J. A. M. van der Mullen, and D. C. Schram, Phys. Rev. Lett. 84, 2622 (2000).

${ }^{33} \mathrm{~S}$. Gerstenkorn and P. Luc, Atlas du Spectroscopie d'Absorption de la Molecule d'Iode (CNRS, Paris, 1978); S. Gerstenkorn and P. Luc, Rev. Phys. Appl. 14, 791 (1979).

${ }^{34}$ H. W. P. van der Heijden, M. G. H. Boogaarts, S. Mazouffre, J. A. M. van der Mullen, and D. C. Schram, Phys. Rev. E 61, 4402 (2000).

${ }^{35}$ S. Mazouffre, M. G. H. Boogaarts, I. S. J. Bakker, P. Vankan, R. Engeln, and D. C. Schram, Phys. Rev. E 64, 016411 (2001).

${ }^{36}$ M. C. M. van de Sanden, J. M. de Regt, G. M. Janssen, J. A. M. van der Mullen, D. C. Schram, and B. van der Sijde, Rev. Sci. Instrum. 63, 3369 (1992).

${ }^{37}$ W. Demtröder, Laser Spectroscopy (Springer-Verlag, Berlin, 1988).

${ }^{38}$ D. Voslamber, Rev. Sci. Instrum. 71, 2334 (2000).

${ }^{39} \mathrm{R}$. Loudon, The Quantum Theory of Light (Oxford University, New York, 1983).

${ }^{40}$ J. E. M. Goldsmith, J. Opt. Soc. Am. B 6, 1979 (1989).

${ }^{41}$ H. R. Griem, Spectral Line Broadening by Plasma (Academic, London, 1974).

${ }^{42}$ S. Bashkin and J. O. Stoner, Jr., Atomic Energy Levels and Grotrian Diagrams (North-Holland, Amsterdam, 1975), Vol. I.

${ }^{43} \mathrm{H}$. Ashkenas and F. S. Sherman, in Proceedings of Rarefied Gasdynamics (Academic, New York, 1966), Vol. 4.

${ }^{44}$ R. Campargue, J. Chem. Phys. 52, 1795 (1970).

${ }^{45}$ Atomic and Molecular Beam Methods, edited by G. Scoles (Oxford University, New York, 1988).

${ }^{46}$ C. I. M. Beenakker, Spectrochim. Acta, Part B 31B, 483 (1976)

${ }^{47}$ M. A. A. Clyne and W. S. Nip, in Reactive Intermediates in the Gas Phase, edited by D. W. Setser (Academic, New York, 1979).

${ }^{48}$ A. McKenzie, M. F. R. Malcahy, and J. R. Steven, J. Chem. Soc., Faraday Trans. 1 70, 549 (1974)

${ }^{49}$ J. C. Miller, Phys. Rev. A 40, 6969 (1989).

${ }^{50}$ R. S. F. Chang, H. Horiguchi, and D. W. Setser, J. Chem. Phys. 73, 778 (1980). 